\title{
Some $q$-Supercongruences from Transformation Formulas for Basic Hypergeometric Series
}

\author{
Victor J. W. Guo ${ }^{1} \cdot$ Michael J. Schlosser ${ }^{2}$ \\ Received: 29 March 2019 / Revised: 15 November 2019 / Accepted: 31 July 2020 / \\ Published online: 30 November 2020 \\ (c) The Author(s) 2020
}

\begin{abstract}
Several new $q$-supercongruences are obtained using transformation formulas for basic hypergeometric series, together with various techniques such as suitably combining terms, and creative microscoping, a method recently developed by the first author in collaboration with Zudilin. More concretely, the results in this paper include $q$ analogues of supercongruences (referring to $p$-adic identities remaining valid for some higher power of $p$ ) established by Long, by Long and Ramakrishna, and several other $q$-supercongruences. The six basic hypergeometric transformation formulas which are made use of are Watson's transformation, a quadratic transformation of Rahman, a cubic transformation of Gasper and Rahman, a quartic transformation of Gasper and Rahman, a double series transformation of Ismail, Rahman and Suslov, and a new transformation formula for a nonterminating very-well-poised ${ }_{12} \phi_{11}$ series. Also, the nonterminating $q$-Dixon summation formula is used. A special case of the new ${ }_{12} \phi_{11}$ transformation formula is further utilized to obtain a generalization of Rogers' linearization formula for the continuous $q$-ultraspherical polynomials.
\end{abstract}

Keywords Basic hypergeometric series · Supercongruences · Identities · Linearization

Communicated by Mourad Ismail.

The first author was partially supported by the National Natural Science Foundation of China (Grant 11771175). The second author was partially supported by Austrian Science Fund Grant P32305.

$\bowtie \quad$ Michael J. Schlosser

michael.schlosser@univie.ac.at

Victor J. W. Guo

jwguo@hytc.edu.cn

1 School of Mathematics and Statistics, Huaiyin Normal University, Huai' an 223300, Jiangsu, People's Republic of China

2 Fakultät für Mathematik, Universität Wien, Oskar-Morgenstern-Platz 1, 1090 Vienna, Austria 
Mathematics Subject Classification Primary 33D15; Secondary 11A07 - 11F33 . 33D45

\section{Introduction}

Ramanujan, in his second letter to Hardy on February 27, 1913, mentioned the following identity

$$
\sum_{k=0}^{\infty}(-1)^{k}(4 k+1) \frac{\left(\frac{1}{2}\right)_{k}^{5}}{k !^{5}}=\frac{2}{\Gamma\left(\frac{3}{4}\right)^{4}}
$$

where $\Gamma(x)$ is the Gamma function and where $(a)_{k}=a(a+1) \cdots(a+k-1)$ is the Pochhammer symbol. A $p$-adic analogue of (1.1) was conjectured by Van Hamme [55, Eq. (A.2)] as follows:

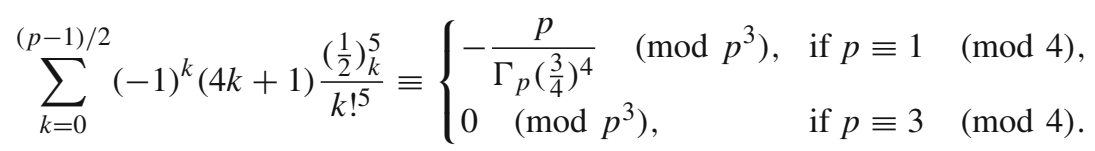

Here and throughout the paper, $p$ always denotes an odd prime and $\Gamma_{p}(x)$ is the $p$-adic Gamma function. The congruence (1.2) was later proved by McCarthy and Osburn [43] through a combination of ordinary and Gaussian hypergeometric series. Recently, the congruence $(1.2)$ for $p \equiv 3(\bmod 4)$ and $p>3$ was further generalized by Liu [37] to the modulus $p^{4}$ case.

It is well known that some truncated hypergeometric series are closely related to Calabi-Yau threefolds over finite fields and are further relevant to the coefficients of modular forms. For example, using the fact that the Calabi-Yau threefold in question is modular, which was proved by Ahlgren and Ono [3], Kilbourn [34] succeeded in proving Van Hamme's (M.2) supercongruence:

$$
\sum_{k=0}^{(p-1) / 2} \frac{\left(\frac{1}{2}\right)_{k}^{4}}{k !^{4}} \equiv a_{p} \quad\left(\bmod p^{3}\right)
$$

where $a_{p}$ is the $p$ th coefficient of a weight 4 modular form

$$
\eta(2 z)^{4} \eta(4 z)^{4}:=q \prod_{n=1}^{\infty}\left(1-q^{2 n}\right)^{4}\left(1-q^{4 n}\right)^{4}, \quad q=e^{2 \pi i z}
$$

Applying Whipple's ${ }_{7} F_{6}$ transformation formula, Long [40] proved that

$$
\sum_{k=0}^{(p-1) / 2}(4 k+1) \frac{\left(\frac{1}{2}\right)_{k}^{6}}{k !^{6}} \equiv p \sum_{k=0}^{(p-1) / 2} \frac{\left(\frac{1}{2}\right)_{k}^{4}}{k !^{4}} \quad\left(\bmod p^{4}\right) \quad \text { for } p>3
$$


which in view of the supercongruence (1.3) can be written as

$$
\sum_{k=0}^{(p-1) / 2}(4 k+1) \frac{\left(\frac{1}{2}\right)_{k}^{6}}{k !^{6}} \equiv p a_{p} \quad\left(\bmod p^{4}\right) \text { for } p>3
$$

The main aim of this paper is to give $q$-analogues of some known supercongruences, including a partial $q$-analogue of Long's supercongruence (1.4) (partial in the sense that the modulo $p^{4}$ condition is replaced by the weaker condition modulo $p^{3}$ ). We provide such a result in Theorem 2.1 in the form of two transformations of truncated basic hypergeometric series. In addition, several other $q$-supercongruences are given. These results are proved by special instances of transformation formulas for basic hypergeometric series. (See Theorem A.1 in the Appendix for a new basic hypergeometric transformation formula which we make use of.)

Throughout we assume $q$ to be fixed with $0<|q|<1$. We refer to $q$ as the "base". For $a, k \in \mathbb{C}$, the $q$-shifted factorial is defined by

$$
(a ; q)_{k}:=\frac{(a ; q)_{\infty}}{\left(a q^{k} ; q\right)_{\infty}}, \quad \text { where } \quad(a ; q)_{\infty}=\prod_{j \geq 0}\left(1-a q^{j}\right) .
$$

For brevity, we frequently use the shorthand notation

$$
\left(a_{1}, \ldots, a_{m} ; q\right)_{k}=\left(a_{1} ; q\right)_{k} \ldots\left(a_{m} ; q\right)_{k}, \quad k \in \mathbb{C} \cup \infty
$$

Moreover, the $q$-binomial coefficients $\left[\begin{array}{l}x \\ k\end{array}\right]$ are defined by

$$
\left[\begin{array}{l}
x \\
k
\end{array}\right]=\left[\begin{array}{l}
x \\
k
\end{array}\right]_{q}= \begin{cases}\frac{\left(q^{x-k+1} ; q\right)_{k}}{(q ; q)_{k}}, & \text { if } k \geq 0 \\
0, & \text { otherwise }\end{cases}
$$

It is easy to see that

$$
(-1)^{k} q^{k^{2}}\left[\begin{array}{c}
-\frac{1}{2} \\
k
\end{array}\right]_{q^{2}}=\frac{\left(q ; q^{2}\right)_{k}}{\left(q^{2} ; q^{2}\right)_{k}}=\frac{1}{(-q ; q)_{k}^{2}}\left[\begin{array}{c}
2 k \\
k
\end{array}\right] .
$$

Following Gasper and Rahman [13], basic hypergeometric ${ }_{r} \phi_{s}$ series with $r$ upper parameters $a_{1}, \ldots, a_{r}, s$ lower parameters $b_{1}, \ldots, b_{s}$, base $q$, and argument $z$ are defined by

$$
{ }_{r} \phi_{s}\left[\begin{array}{c}
a_{1}, a_{2}, \ldots, a_{r} \\
b_{1}, \ldots, b_{s}
\end{array} ; q, z\right]:=\sum_{k=0}^{\infty} \frac{\left(a_{1}, a_{2}, \ldots, a_{r} ; q\right)_{k}}{\left(q, b_{1}, \ldots, b_{s} ; q\right)_{k}}\left[(-1)^{k} q^{\left(\begin{array}{c}
k \\
2
\end{array}\right)}\right]^{1+s-r} z^{k}
$$

where $q \neq 0$ when $r>s+1$. Such a series terminates if one of the upper parameters, say, $a_{r}$, is of the form $q^{-n}$, where $n$ is a nonnegative integer. If the series does not terminate, then it converges for $|z|<1$. 
In many of our proofs we will make use of Watson's ${ }_{8} \phi_{7}$ transformation formula [13, Appendix (III.17)]:

$$
\begin{aligned}
{ }_{8} \phi_{7}\left[\begin{array}{c}
a, q a^{\frac{1}{2}},-q a^{\frac{1}{2}}, \quad b, \quad c, \quad d, \quad e, \quad f \\
a^{\frac{1}{2}},-a^{\frac{1}{2}}, a q / b, a q / c, a q / d, a q / e, a q / f
\end{array} ;, \frac{a^{2} q^{2}}{b c d e f}\right] \\
\quad=\frac{(a q, a q / d e, a q / d f, a q / e f ; q)_{\infty}}{(a q / d, a q / e, a q / f, a q / \operatorname{def} ; q)_{\infty}} 4 \phi_{3}\left[\begin{array}{c}
a q / b c, d, e, f \\
a q / b, a q / c, d e f / a
\end{array} ; q, q\right],
\end{aligned}
$$

which is valid whenever the ${ }_{8} \phi_{7}$ series converges and the ${ }_{4} \phi_{3}$ series terminates. In particular, we will also make use of the limiting case $f=q^{-n} \rightarrow \infty$, which we state for convenience:

$$
\begin{aligned}
& \sum_{k=0}^{\infty} \frac{(-1)^{k}\left(1-a q^{2 k}\right)(a, b, c, d, e ; q)_{k}}{(1-a)(q, a q / b, a q / c, a q / d, a q / e ; q)_{k}}\left(\frac{a^{2} q^{2}}{b c d e}\right)^{k} q^{\left(\begin{array}{c}
k \\
2
\end{array}\right)} \\
& \quad=\frac{(a q, a q / d e ; q)_{\infty}}{(a q / d, a q / e ; q)_{\infty}}{ }_{3} \phi_{2}\left[\begin{array}{c}
a q / b c, d, e \\
a q / b, a q / c
\end{array} ;, \frac{a q}{d e}\right] .
\end{aligned}
$$

Other transformations we make use of are a quadratic transformation formula of Rahman, stated in (6.3), a cubic transformation formula of Gasper and Rahman, stated in (7.1), a quartic transformation formula by Gasper and Rahman, stated in (8.1), a double series transformation by Ismail, Rahman and Suslov, stated in (11.1), and a new transformation formula for a nonterminating ${ }_{12} \phi_{11}$ series into two multiples of nonterminating ${ }_{4} \phi_{3}$ series, given as Theorem A.1 in the Appendix. We also make use of the $q$-Dixon summation, stated in (10.1).

For further material on basic hypergeometric series and more generally, on special functions, we refer to the text books by Gasper and Rahman [13], and by Andrews, Askey and Roy [2], respectively. In particular, in our computations we implicitly make heavy use of elementary manipulations of $q$-shifted factorials (see [13, Appendix I]).

Recall that the $q$-integer is defined as $[n]=[n]_{q}=1+q+\cdots+q^{n-1}$. Moreover, the $n$th cyclotomic polynomial $\Phi_{n}(q)$ is given by

$$
\Phi_{n}(q):=\prod_{\substack{1 \leq k \leq n \\ \operatorname{gcd}(n, k)=1}}\left(q-\zeta^{k}\right),
$$

where $\zeta$ is an $n$th primitive root of unity. It is clear that $\Phi_{n}(q)$ is a polynomial in $q$ with integer coefficients. Further,

$$
\prod_{d \mid n, d>1} \Phi_{d}(q)=[n]
$$

in particular, $\Phi_{p}(q)=[p]$ for any prime $p$.

We say that two rational functions $A(q)$ and $B(q)$ in $q$ are congruent modulo a polynomial $P(q)$, denoted by $A(q) \equiv B(q)(\bmod P(q))$, if the numerator of the reduced form of $A(q)-B(q)$ is divisible by $P(q)$ in the polynomial ring $\mathbb{Z}[q]$. 
We refer the reader to $[1,7,16-24,27,36,39,45,49,53,54,57,58]$ for some interesting $q$-congruences.

\section{The Main Results}

The following is our $q$-analogue of (1.4), where the modulo $p^{4}$ condition is replaced by the weaker condition modulo $p^{3}$.

Theorem 2.1 Let $n$ be a positive odd integer. Then

$$
\sum_{k=0}^{n-1}[4 k+1] \frac{\left(q ; q^{2}\right)_{k}^{6}}{\left(q^{2} ; q^{2}\right)_{k}^{6}} q^{k} \equiv[n] q^{(1-n) / 2} \sum_{k=0}^{(n-1) / 2} \frac{\left(q ; q^{2}\right)_{k}^{4}}{\left(q^{2} ; q^{2}\right)_{k}^{4}} q^{2 k} \quad\left(\bmod [n] \Phi_{n}(q)^{2}\right),
$$

and

$$
\sum_{k=0}^{(n-1) / 2}[4 k+1] \frac{\left(q ; q^{2}\right)_{k}^{6}}{\left(q^{2} ; q^{2}\right)_{k}^{6}} q^{k} \equiv[n] q^{(1-n) / 2} \sum_{k=0}^{(n-1) / 2} \frac{\left(q ; q^{2}\right)_{k}^{4}}{\left(q^{2} ; q^{2}\right)_{k}^{4}} q^{2 k} \quad\left(\bmod [n] \Phi_{n}(q)^{2}\right)
$$

Noticing that the terms corresponding to $k$ in the upper half range $(n-1) / 2<k \leq n-1$ are congruent to 0 modulo $\Phi_{n}(q)^{3}$ but not modulo $[n] \Phi_{n}(q)^{2}$ in general, we conclude that (2.1a) and (2.1b) are in fact different congruences. Of course, when $n=p$ is an odd prime and $q=1$, they are both equivalent to (1.4) modulo $p^{3}$. The proof of Theorem 2.1 is deferred to Sect. 3.

Van Hamme [55, Eq. (H.2)] proved the following supercongruence:

$$
\sum_{k=0}^{(p-1) / 2} \frac{\left(\frac{1}{2}\right)_{k}^{3}}{k !^{3}} \equiv\left\{\begin{array}{lll}
-\Gamma_{p}\left(\frac{1}{4}\right)^{4}\left(\bmod p^{2}\right), & \text { if } p \equiv 1 & (\bmod 4) \\
0 \quad\left(\bmod p^{2}\right), & \text { if } p \equiv 3 & (\bmod 4)
\end{array}\right.
$$

The first author and Zeng [27, Cor. 1.2] gave a $q$-analogue of (2.2) as follows:

$$
\begin{aligned}
& \sum_{k=0}^{(p-1) / 2} \frac{\left(q ; q^{2}\right)_{k}^{2}\left(q^{2} ; q^{4}\right)_{k}}{\left(q^{2} ; q^{2}\right)_{k}^{2}\left(q^{4} ; q^{4}\right)_{k}} q^{2 k}
\end{aligned}
$$

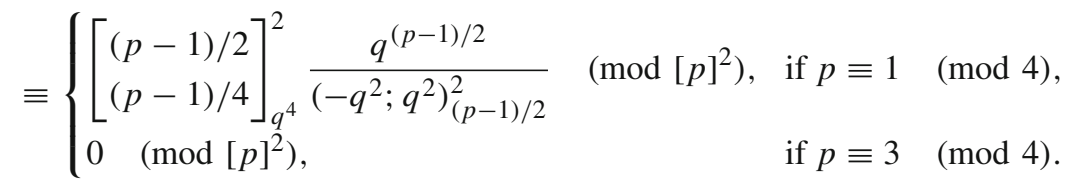

We do not know any $q$-analogue of (1.2). However, we are able to provide a $q$-analogue of a very closely related congruence. In particular, since $\Gamma_{p}\left(\frac{1}{4}\right)^{4} \Gamma_{p}\left(\frac{3}{4}\right)^{4}=1$, from 
(1.2) and (2.2) we deduce that

$$
\sum_{k=0}^{(p-1) / 2}(-1)^{k}(4 k+1) \frac{\left(\frac{1}{2}\right)_{k}^{5}}{k !^{5}} \equiv p \sum_{k=0}^{(p-1) / 2} \frac{\left(\frac{1}{2}\right)_{k}^{3}}{k !^{3}} \quad\left(\bmod p^{3}\right)
$$

which was already noticed by Mortenson [44]. We are able to give the following complete $q$-analogue of (2.3).

Theorem 2.2 Let $n$ be a positive odd integer. Then

$$
\begin{aligned}
& \sum_{k=0}^{n-1}(-1)^{k}[4 k+1] \frac{\left(q ; q^{2}\right)_{k}^{5}}{\left(q^{2} ; q^{2}\right)_{k}^{5}} q^{k^{2}+k} \\
& \quad \equiv[n] q^{(1-n) / 2} \sum_{k=0}^{(n-1) / 2} \frac{\left(q ; q^{2}\right)_{k}^{3}}{\left(q^{2} ; q^{2}\right)_{k}^{3}} q^{2 k} \quad\left(\bmod [n] \Phi_{n}(q)^{2}\right),
\end{aligned}
$$

and

$$
\begin{aligned}
& \sum_{k=0}^{(n-1) / 2}(-1)^{k}[4 k+1] \frac{\left(q ; q^{2}\right)_{k}^{5}}{\left(q^{2} ; q^{2}\right)_{k}^{5}} q^{k^{2}+k} \\
& \equiv[n] q^{(1-n) / 2} \sum_{k=0}^{(n-1) / 2} \frac{\left(q ; q^{2}\right)_{k}^{3}}{\left(q^{2} ; q^{2}\right)_{k}^{3}} q^{2 k} \quad\left(\bmod [n] \Phi_{n}(q)^{2}\right)
\end{aligned}
$$

Note that, just like in Theorem 2.1, the two congruences (2.4a) and (2.4b) are not equivalent. The proof of Theorem 2.2 is deferred to Sect. 3 .

Long and Ramakrishna [41, Thm. 2] proved the following supercongruence:

$$
\sum_{k=0}^{p-1}(6 k+1) \frac{\left(\frac{1}{3}\right)_{k}^{6}}{k !^{6}} \equiv\left\{\begin{array}{lll}
-p \Gamma_{p}\left(\frac{1}{3}\right)^{9}\left(\bmod p^{6}\right), & \text { if } p \equiv 1 \quad(\bmod 6) \\
-\frac{10 p^{4}}{27} \Gamma_{p}\left(\frac{1}{3}\right)^{9}\left(\bmod p^{6}\right), & \text { if } p \equiv 5 \quad(\bmod 6)
\end{array}\right.
$$

This result is stronger than Van Hamme's (D.2) supercongruence conjecture which asserts a congruence modulo $p^{4}$ for $p \equiv 1(\bmod 6)$. Long and Ramakrishna also pointed out that (2.5) does not hold modulo $p^{7}$ in general.

We propose the following partial $q$-analogue of Long and Ramakrishna's supercongruence (2.5).

Theorem 2.3 Let $n$ be a positive integer coprime with 3. Then

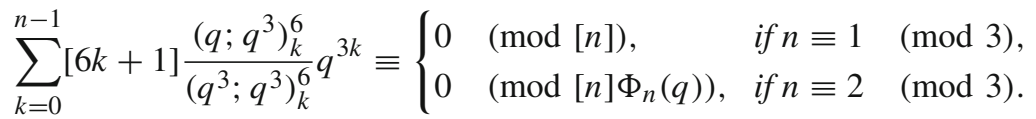


We also partially confirm the $a=1$ case of the second congruence in [28, Conj. 5.2].

Theorem 2.4 Let $d$ and $n$ be positive integers with $d>2$ and $n \equiv-1(\bmod d)$. Then

$$
\sum_{k=0}^{n-1}[2 d k+1] \frac{\left(q ; q^{d}\right)_{k}^{4}}{\left(q^{d} ; q^{d}\right)_{k}^{4}} q^{(d-2) k} \equiv 0 \quad\left(\bmod \Phi_{n}(q)^{2}\right) .
$$

The proofs of Theorems 2.3 and 2.4 are deferred to Sect. 4.

In Sect. 3, we shall prove Theorems 2.1 and 2.2 using the creative microscoping method developed by the first author and Zudilin [28]. Roughly speaking, to prove a $q$-supercongruence modulo $\Phi_{n}(q)^{3}$, we prove its generalization with an extra parameter $a$ so that the corresponding congruence holds modulo $\Phi_{n}(q)\left(1-a q^{n}\right)\left(a-q^{n}\right)$. Since the polynomials $\Phi_{n}(q), 1-a q^{n}$, and $a-q^{n}$ are pairwise relatively prime, this generalized $q$-congruence can be established modulo these three polynomials individually. Finally, by taking the limit $a \rightarrow 1$, we obtain the original $q$-supercongruence of interest. We learned that this creative microscoping method has already caught the interests of Guillera [14] and Straub [50].

Further, we introduce a new idea for proving some congruences modulo $\Phi_{n}(q)$. In many instances in this paper, the congruences $\sum_{k=0}^{(n-1) / 2} a_{n, k} \equiv 0\left(\bmod \Phi_{n}(q)\right)$ are proved by simply showing $a_{k}+a_{(n-1) / 2-k} \equiv 0\left(\bmod \Phi_{n}(q)\right)$ (instead of, say, evaluating certain infinite series at roots of unity which was illustrated in [28]).

The proofs of Theorems 2.3 and 2.4 in Sect. 4 again are done by showing a more general identity but otherwise are accomplished in a slightly different way. All the proofs of Theorems 2.1-2.4 in Sects. 3 and 4, and of the further results from Sect. 5, are based on Watson's ${ }_{8} \phi_{7}$ transformation formula. We also confirm a three-parametric $q$-congruence conjecture in Sect. 6 based on a quadratic transformation formula of Rahman. Further, in Sect. 7 we deduce some $q$-congruences from a cubic transformation formula of Gasper and Rahman. Similarly, in Sect. 8 we deduce some $q$-congruences from a quartic transformation formula of Gasper and Rahman. The $q$-supercongruences in Sect. 9 are proved similarly but are derived using a new ${ }_{12} \phi_{11}$ transformation formula. Since the latter formula is of independent interest, its derivation is given in the Appendix. It is also shown there how a special case of the ${ }_{12} \phi_{11}$ transformation formula can be utilized to obtain a generalization of Rogers' linearization formula for the continuous $q$-ultraspherical polynomials. In Sect. 10 some $q$-supercongruences are deduced from the $q$-Dixon summation. In Sect. 11 we deduce $q$-super congruences - most of them only conjectural—-from a double series transformation of Ismail, Rahman and Suslov. Finally, in Sect. 12, some concluding remarks are given and some related conjectures for further study are proposed. For example, we conjecture that the congruence (2.6) still holds modulo $[n] \Phi_{n}(q)^{3}$ for $n \equiv 2(\bmod 3)$.

\section{Proofs of Theorems 2.1 and 2.2}

We first give the following lemma. 
Lemma 3.1 Let $n$ be a positive odd integer. Then, for $0 \leq k \leq(n-1) / 2$, we have

$$
\frac{\left(a q ; q^{2}\right)_{(n-1) / 2-k}}{\left(q^{2} / a ; q^{2}\right)_{(n-1) / 2-k}} \equiv(-a)^{(n-1) / 2-2 k} \frac{\left(a q ; q^{2}\right)_{k}}{\left(q^{2} / a ; q^{2}\right)_{k}} q^{(n-1)^{2} / 4+k} \quad\left(\bmod \Phi_{n}(q)\right)
$$

Proof Since $q^{n} \equiv 1\left(\bmod \Phi_{n}(q)\right)$, we have

$$
\begin{aligned}
\frac{\left(a q ; q^{2}\right)_{(n-1) / 2}}{\left(q^{2} / a ; q^{2}\right)_{(n-1) / 2}} & =\frac{(1-a q)\left(1-a q^{3}\right) \cdots\left(1-a q^{n-2}\right)}{\left(1-q^{2} / a\right)\left(1-q^{4} / a\right) \cdots\left(1-q^{n-1} / a\right)} \\
& \equiv \frac{(1-a q)\left(1-a q^{3}\right) \cdots\left(1-a q^{n-2}\right)}{\left(1-q^{2-n} / a\right)\left(1-q^{4-n} / a\right) \cdots\left(1-q^{-1} / a\right)} \\
& =(-a)^{(n-1) / 2} q^{(n-1)^{2} / 4} \quad\left(\bmod \Phi_{n}(q)\right) .
\end{aligned}
$$

Further, modulo $\Phi_{n}(q)$, we have

$$
\begin{aligned}
& \frac{\left(a q ; q^{2}\right)_{(n-1) / 2-k}}{\left(q^{2} / a ; q^{2}\right)_{(n-1) / 2-k}} \\
& =\frac{\left(a q ; q^{2}\right)_{(n-1) / 2}}{\left(q^{2} / a ; q^{2}\right)_{(n-1) / 2}} \frac{\left(1-q^{n+1-2 k} / a\right)\left(1-q^{n+3-2 k} / a\right) \cdots\left(1-q^{n-1} / a\right)}{\left(1-a q^{n-2 k}\right)\left(1-a q^{n+2-2 k}\right) \cdots\left(1-a q^{n-2}\right)} \\
& \equiv \frac{\left(a q ; q^{2}\right)_{(n-1) / 2}}{\left(q^{2} / a ; q^{2}\right)_{(n-1) / 2}} \frac{\left(1-q^{1-2 k} / a\right)\left(1-q^{3-2 k} / a\right) \cdots\left(1-q^{-1} / a\right)}{\left(1-a q^{-2 k}\right)\left(1-a q^{2-2 k}\right) \cdots\left(1-a q^{-2}\right)},
\end{aligned}
$$

which in combination with (3.1) establishes the assertion.

We now use the above lemma to prove the following result which was originally conjectured by the first author and Zudilin [28, Conj. 5.6].

Theorem 3.2 Let $n \equiv 3(\bmod 4)$ be a positive integer. Then

$$
\sum_{k=0}^{(n-1) / 2} \frac{\left(a q, q / a ; q^{2}\right)_{k}\left(q^{2} ; q^{4}\right)_{k}}{\left(a q^{2}, q^{2} / a ; q^{2}\right)_{k}\left(q^{4} ; q^{4}\right)_{k}} q^{2 k} \equiv 0 \quad\left(\bmod \Phi_{n}(q)\right)
$$

Proof By Lemma 3.1, we have

$$
\frac{\left(a q, q / a ; q^{2}\right)_{(n-1) / 2-k}}{\left(a q^{2}, q^{2} / a ; q^{2}\right)_{(n-1) / 2-k}} \equiv \frac{\left(a q, q / a ; q^{2}\right)_{k}}{\left(a q^{2}, q^{2} / a ; q^{2}\right)_{k}} q^{(n-1)^{2} / 2+2 k} \quad\left(\bmod \Phi_{n}(q)\right),
$$

and

$$
\frac{\left(q^{2} ; q^{4}\right)_{(n-1) / 2-k}}{\left(q^{4} ; q^{4}\right)_{(n-1) / 2-k}} \equiv(-1)^{(n-1) / 2-2 k} \frac{\left(q^{2} ; q^{4}\right)_{k}}{\left(q^{4} ; q^{4}\right)_{k}} q^{(n-1)^{2} / 2+2 k} \quad\left(\bmod \Phi_{n}\left(q^{2}\right)\right) .
$$


Noticing that $q^{n} \equiv 1\left(\bmod \Phi_{n}(q)\right)$ and, for odd $n, \Phi_{n}\left(q^{2}\right)=\Phi_{n}(q) \Phi_{n}(-q)$, we get

$$
\begin{aligned}
& \frac{\left(a q, q / a ; q^{2}\right)_{(n-1) / 2-k}\left(q^{2} ; q^{4}\right)_{(n-1) / 2-k} q^{n-1-2 k}}{\left(a q^{2}, q^{2} / a ; q^{2}\right)_{(n-1) / 2-k}\left(q^{4} ; q^{4}\right)_{(n-1) / 2-k}} \\
& \equiv-\frac{\left(a q, q / a ; q^{2}\right)_{k}\left(q^{2} ; q^{4}\right)_{k} q^{2 k}}{\left(a q^{2}, q^{2} / a ; q^{2}\right)_{k}\left(q^{4} ; q^{4}\right)_{k}} \quad\left(\bmod \Phi_{n}(q)\right)
\end{aligned}
$$

for any positive integer $n$ with $n \equiv 3(\bmod 4)$ and $0 \leq k \leq(n-1) / 2$. This completes the proof of the theorem.

Similarly, we can prove that the third $q$-congruence in [28, Conj. 5.2] is true modulo $\Phi_{n}(q)$ and is therefore further true modulo [ $\left.n\right]$ (again as in the proof of Theorem 2.1).

We shall establish the following two-parameter generalization of Theorem 2.1.

Theorem 3.3 Let $n$ be a positive odd integer. Then, modulo $\Phi_{n}(q)\left(1-a q^{n}\right)\left(a-q^{n}\right)$,

$$
\begin{aligned}
& \sum_{k=0}^{(n-1) / 2}[4 k+1] \frac{\left(a q, q / a, b q ; q^{2}\right)_{k}\left(q ; q^{2}\right)_{k}^{3}}{\left(a q^{2}, q^{2} / a, q^{2} / b ; q^{2}\right)_{k}\left(q^{2} ; q^{2}\right)_{k}^{3}}\left(\frac{q}{b}\right)^{k} \\
& \equiv[n] q^{(1-n) / 2} \sum_{k=0}^{(n-1) / 2} \frac{\left(a q, q / a, q / b, q ; q^{2}\right)_{k}}{\left(q^{2} / b ; q^{2}\right)_{k}\left(q^{2} ; q^{2}\right)_{k}^{3}} q^{2 k}
\end{aligned}
$$

Proof For $a=q^{-n}$ or $a=q^{n}$, the left-hand side of (3.2) is equal to

$$
\begin{aligned}
& \sum_{k=0}^{(n-1) / 2}[4 k+1] \frac{\left(q^{1-n}, q^{1+n}, b q ; q^{2}\right)_{k}\left(q ; q^{2}\right)_{k}^{3}}{\left(q^{2-n}, q^{2+n}, q^{2} / b ; q^{2}\right)_{k}\left(q^{2} ; q^{2}\right)_{k}^{3}}\left(\frac{q}{b}\right)^{k} \\
& \quad={ }_{8} \phi_{7}\left[\begin{array}{r}
q, q^{\frac{5}{2}},-q^{\frac{5}{2}}, \quad b q, \quad q, q^{1-n}, q^{1+n}, q \\
\left.q^{\frac{1}{2}},-q^{\frac{1}{2}}, q^{2} / b, q^{2}, q^{2+n}, q^{2-n}, q^{2} ; q^{2}, \frac{q}{b}\right]
\end{array}\right.
\end{aligned}
$$

By Watson's ${ }_{8} \phi_{7}$ transformation formula (1.7), we can rewrite the right-hand side of (3.3) as

$$
\begin{aligned}
& \lim _{z \rightarrow 1} \frac{\left(q^{3}, q, q^{1+n}, z q^{1-n} ; q^{2}\right)_{\infty}}{\left(q^{2+n}, q^{2-n}, q^{2}, z ; q^{2}\right)_{\infty}} 4 \phi_{3}\left[\begin{array}{c}
q / b, q^{1-n}, q^{1+n}, q \\
q^{2}, q^{2} / b, q^{2}
\end{array} q^{2}, q^{2}\right] \\
& =\frac{\left(q ; q^{2}\right)_{(n+1) / 2}\left(q^{1-n} ; q^{2}\right)_{(n-1) / 2}}{\left(q^{2-n} ; q^{2}\right)_{(n+1) / 2}\left(q^{2} ; q^{2}\right)_{(n-1) / 2}} \sum_{k=0}^{(n-1) / 2} \frac{\left(q^{1-n}, q^{1+n}, q / b, q ; q^{2}\right)_{k}}{\left(q^{2} / b ; q^{2}\right)_{k}\left(q^{2} ; q^{2}\right)_{k}^{3}} q^{2 k} \text {. }
\end{aligned}
$$

It is easy to see that the fraction before the sum on the right-hand side of (3.4) is equal to $[n] q^{(1-n) / 2}$. This proves that the congruence (3.2) holds modulo $1-a q^{n}$ or $a-q^{n}$. 
Moreover, by Lemma 3.1, it is easy to see that, modulo $\Phi_{n}(q)$, the $k$ th and $((n-$ $1) / 2-k$ )th terms on the left-hand side of (3.2) cancel each other, i.e.,

$$
\begin{gathered}
\frac{[2 n-4 k-1]\left(a q, q / a, b q ; q^{2}\right)_{(n-1) / 2-k}\left(q ; q^{2}\right)_{(n-1) / 2-k}^{3}}{\left(a q^{2}, q^{2} / a, q^{2} / b ; q^{2}\right)_{(n-1) / 2-k}\left(q^{2} ; q^{2}\right)_{(n-1) / 2-k}^{3}}\left(\frac{q}{b}\right)^{(n-1) / 2-k} \\
\equiv-[4 k+1] \frac{\left(a q, q / a, b q ; q^{2}\right)_{k}\left(q ; q^{2}\right)_{k}^{3}}{\left(a q^{2}, q^{2} / a, q^{2} / b ; q^{2}\right)_{k}\left(q^{2} ; q^{2}\right)_{k}^{3}}\left(\frac{q}{b}\right)^{k} \quad\left(\bmod \Phi_{n}(q)\right) .
\end{gathered}
$$

When the left-hand side of (3.2) has an odd number of factors, the central term will remain. This happens when $n=4 l+1$ for some positive integer $l$, and in this case the central term has index $k=l$ and one directly sees that $[4 k+1]=[n]$ is a factor of the summand. In total, this proves that the left-hand side of (3.2) is congruent to 0 modulo $\Phi_{n}(q)$, and therefore the congruence (3.2) also holds modulo $\Phi_{n}(q)$. Since $\Phi_{n}(q), 1-a q^{n}$ and $a-q^{n}$ are pairwise relatively prime polynomials, the proof of (3.2) is complete.

Proof of Theorem 2.1 The limits of the denominators on both sides of (3.2) as $a \rightarrow 1$ are relatively prime to $\Phi_{n}(q)$, since $0 \leq k \leq(n-1) / 2$. On the other hand, the limit of $\left(1-a q^{n}\right)\left(a-q^{n}\right)$ as $a \rightarrow 1$ has the factor $\Phi_{n}(q)^{2}$. Thus, the limiting case $a, b \rightarrow 1$ of (3.2) gives the following congruence

$$
\sum_{k=0}^{(n-1) / 2}[4 k+1] \frac{\left(q ; q^{2}\right)_{k}^{6}}{\left(q^{2} ; q^{2}\right)_{k}^{6}} q^{k} \equiv[n] q^{(1-n) / 2} \sum_{k=0}^{(n-1) / 2} \frac{\left(q ; q^{2}\right)_{k}^{4}}{\left(q^{2} ; q^{2}\right)_{k}^{4}} q^{2 k}\left(\bmod \Phi_{n}(q)^{3}\right)
$$

which also implies that

$$
\sum_{k=0}^{n-1}[4 k+1] \frac{\left(q ; q^{2}\right)_{k}^{6}}{\left(q^{2} ; q^{2}\right)_{k}^{6}} q^{k} \equiv[n] q^{(1-n) / 2} \sum_{k=0}^{(n-1) / 2} \frac{\left(q ; q^{2}\right)_{k}^{4}}{\left(q^{2} ; q^{2}\right)_{k}^{4}} q^{2 k}\left(\bmod \Phi_{n}(q)^{3}\right)
$$

since $\left(q ; q^{2}\right)_{k}^{6} /\left(q^{2} ; q^{2}\right)_{k}^{6} \equiv 0\left(\bmod \Phi_{n}(q)^{3}\right)$ for $k$ in the range $(n-1) / 2<k \leq$ $n-1$. It remains to show that the above two congruences are still true modulo $[n]$, or equivalently,

$$
\sum_{k=0}^{(n-1) / 2}[4 k+1] \frac{\left(q ; q^{2}\right)_{k}^{6}}{\left(q^{2} ; q^{2}\right)_{k}^{6}} q^{k} \equiv 0 \quad(\bmod [n])
$$

and

$$
\sum_{k=0}^{n-1}[4 k+1] \frac{\left(q ; q^{2}\right)_{k}^{6}}{\left(q^{2} ; q^{2}\right)_{k}^{6}} q^{k} \equiv 0 \quad(\bmod [n])
$$

For $n>1$, let $\zeta \neq 1$ be an $n$th root of unity, not necessarily primitive. That is, $\zeta$ is a primitive root of unity of odd degree $d \mid n$. Let $c_{q}(k)$ denote the $k$ th term on the 
left-hand side of the congruences in (3.7), i.e.,

$$
c_{q}(k)=[4 k+1] \frac{\left(q ; q^{2}\right)_{k}^{6}}{\left(q^{2} ; q^{2}\right)_{k}^{6}} q^{k}=[4 k+1]\left[\begin{array}{c}
2 k \\
k
\end{array}\right]^{6} \frac{q^{k}}{(-q ; q)_{k}^{12}} .
$$

The congruences (3.5) and (3.6) with $n=d$ imply that

$$
\sum_{k=0}^{(d-1) / 2} c_{\zeta}(k)=\sum_{k=0}^{d-1} c_{\zeta}(k)=0
$$

Observe that

$$
\frac{c_{\zeta}(\ell d+k)}{c_{\zeta}(\ell d)}=\lim _{q \rightarrow \zeta} \frac{c_{q}(\ell d+k)}{c_{q}(\ell d)}=c_{\zeta}(k)
$$

We have

$$
\sum_{k=0}^{n-1} c_{\zeta}(k)=\sum_{\ell=0}^{n / d-1} \sum_{k=0}^{d-1} c_{\zeta}(\ell d+k)=\sum_{\ell=0}^{n / d-1} c_{\zeta}(\ell d) \sum_{k=0}^{d-1} c_{\zeta}(k)=0
$$

and

$$
\sum_{k=0}^{(n-1) / 2} c_{\zeta}(k)=\sum_{\ell=0}^{(n / d-3) / 2} c_{\zeta}(\ell d) \sum_{k=0}^{d-1} c_{\zeta}(k)+\sum_{k=0}^{(d-1) / 2} c_{\zeta}((n-d) / 2+k)=0
$$

which means that the sums $\sum_{k=0}^{n-1} c_{q}(k)$ and $\sum_{k=0}^{(n-1) / 2} c_{q}(k)$ are both divisible by the cyclotomic polynomial $\Phi_{d}(q)$. Since this is true for any divisor $d>1$ of $n$, we conclude that they are divisible by

$$
\prod_{d \mid n, d>1} \Phi_{d}(q)=[n]
$$

thus establishing (3.7).

Proof of Theorem 2.2 Similarly as in the proof of Theorem 2.1, letting $a \rightarrow 1$ and $b \rightarrow \infty$ in (3.2), we obtain

$$
\sum_{k=0}^{(n-1) / 2}(-1)^{k}[4 k+1] \frac{\left(q ; q^{2}\right)_{k}^{5}}{\left(q^{2} ; q^{2}\right)_{k}^{5}} q^{k^{2}+k} \equiv[n] q^{(1-n) / 2} \sum_{k=0}^{(n-1) / 2} \frac{\left(q ; q^{2}\right)_{k}^{3}}{\left(q^{2} ; q^{2}\right)_{k}^{3}} q^{2 k} \quad\left(\bmod \Phi_{n}(q)^{3}\right)
$$

which also implies that

$$
\sum_{k=0}^{n-1}(-1)^{k}[4 k+1] \frac{\left(q ; q^{2}\right)_{k}^{5}}{\left(q^{2} ; q^{2}\right)_{k}^{5}} q^{k^{2}+k} \equiv[n] q^{(1-n) / 2} \sum_{k=0}^{(n-1) / 2} \frac{\left(q ; q^{2}\right)_{k}^{3}}{\left(q^{2} ; q^{2}\right)_{k}^{3}} q^{2 k} \quad\left(\bmod \Phi_{n}(q)^{3}\right) .
$$


Along the same lines as in the proof of Theorem 2.1, we can show that

$$
\begin{aligned}
& \sum_{k=0}^{n-1}(-1)^{k}[4 k+1] \frac{\left(q ; q^{2}\right)_{k}^{5}}{\left(q^{2} ; q^{2}\right)_{k}^{5}} q^{k^{2}+k} \\
& \equiv \sum_{k=0}^{(n-1) / 2}(-1)^{k}[4 k+1] \frac{\left(q ; q^{2}\right)_{k}^{5}}{\left(q^{2} ; q^{2}\right)_{k}^{5}} q^{k^{2}+k} \equiv 0 \quad(\bmod [n])
\end{aligned}
$$

Combining the above congruences, we are led to (2.4a) and (2.4b).

\section{Proofs of Theorems 2.3 and 2.4}

We shall prove the following common generalization of Theorems 2.3 and 2.4.

Theorem 4.1 Let $n$ and $d$ be positive integers with $d \geq 3$ and $\operatorname{gcd}(n, d)=1$. Then

$$
\begin{aligned}
& \sum_{k=0}^{n-1}[2 d k+1] \frac{\left(a q, q / a ; q^{d}\right)_{k}\left(q ; q^{d}\right)_{k}^{4}}{\left(a q^{d}, q^{d} / a ; q^{d}\right)_{k}\left(q^{d} ; q^{d}\right)_{k}^{4}} q^{(2 d-3) k} \\
& \equiv\left\{\begin{array}{lll}
0 & \left(\bmod \Phi_{n}(q)^{2}\right), & \text { if } n \equiv-1 \quad(\bmod d), \\
0 & \left(\bmod \Phi_{n}(q)\right), & \text { otherwise }
\end{array}\right.
\end{aligned}
$$

Proof Let $\alpha$ and $j$ be integers. Since

$$
\left(1-q^{\alpha n-d j+d-1}\right)\left(1-q^{\alpha n+d j-d+1}\right)+\left(1-q^{d j-d+1}\right)^{2} q^{\alpha n-d j+d-1}=\left(1-q^{\alpha n}\right)^{2}
$$

and $1-q^{\alpha n} \equiv 0\left(\bmod \Phi_{n}(q)\right)$, we obtain

$$
\left(1-q^{\alpha n-d j+d-1}\right)\left(1-q^{\alpha n+d j-d+1}\right) \equiv-\left(1-q^{d j-d+1}\right)^{2} q^{\alpha n-d j+d-1} \quad\left(\bmod \Phi_{n}(q)^{2}\right) .
$$

It follows that

$$
\left(q^{1-\alpha n}, q^{1+\alpha n} ; q^{d}\right)_{k} \equiv\left(q ; q^{d}\right)_{k}^{2} \quad\left(\bmod \Phi_{n}(q)^{2}\right)
$$

Similarly, we have

$$
\left(q^{d-\alpha n}, q^{d+\alpha n} ; q^{d}\right)_{k} \equiv\left(q^{d} ; q^{d}\right)_{k}^{2} \quad\left(\bmod \Phi_{n}(q)^{2}\right) .
$$

Since $\operatorname{gcd}(n, d)=1$, we know that there exists a positive integer $\alpha<d$ such that $\alpha n \equiv 1(\bmod d)$. Then by [13, Appendix (III.18)] (i.e., (1.7) with $\left.f=q^{1-\alpha n}\right)$, modulo $\Phi_{n}(q)^{2}$, the left-hand side of (4.1) is congruent to

$$
\sum_{k=0}^{(\alpha n-1) / d}[2 d k+1] \frac{\left(a q, q / a, q, q, q^{1+\alpha n}, q^{1-\alpha n} ; q^{d}\right)_{k}}{\left(a q^{d}, q^{d} / a, q^{d}, q^{d}, q^{d-\alpha n}, q^{d+\alpha n} ; q^{d}\right)_{k}} q^{(2 d-3) k}
$$




$$
=\frac{\left(q^{d+1}, q^{d-\alpha n-1} ; q^{d}\right)_{(\alpha n-1) / d}}{\left(q^{d}, q^{d-\alpha n} ; q^{d}\right)_{(\alpha n-1) / d}} 4 \phi_{3}\left[\begin{array}{c}
q^{d-1}, q, q^{1+\alpha n}, q^{1-\alpha n} \\
q^{2}, a q^{d}, q^{d} / a,
\end{array} ; q^{d}, q^{d}\right] .
$$

It is clear that $\left(q^{d+1} ; q^{d}\right)_{(\alpha n-1) / d}$ in the numerator has the factor $1-q^{\alpha n}$ and is therefore divisible by $\Phi_{n}(q)$, while the denominator is coprime with $\Phi_{n}(q)$. This proves (4.1) for the second case.

Furthermore, if $n \equiv-1(\bmod d)$, then, modulo $\Phi_{n}(q)^{2}$, the left-hand side of (4.1) is congruent to

$$
\begin{aligned}
& \quad \sum_{k=0}^{((d-1) n-1) / d}[2 d k+1] \frac{\left(a q, q / a, q, q, q^{1+(d-1) n}, q^{1-(d-1) n} ; q^{d}\right)_{k}}{\left(a q^{d}, q^{d} / a, q^{d}, q^{d}, q^{d-(d-1) n}, q^{d+(d-1) n} ; q^{d}\right) k} q^{(2 d-3) k} \\
& =\frac{\left(q^{d+1}, q^{d-(d-1) n-1} ; q^{d}\right)_{((d-1) n-1) / d}}{\left(q^{d}, q^{d-(d-1) n} ; q^{d}\right)_{((d-1) n-1) / d}} \phi_{3}\left[\begin{array}{c}
\left.q^{d-1}, q, q^{1+(d-1) n}, q^{1-(d-1) n} ; q^{d}, q^{d}\right] \\
q^{2}, a q^{d}, q^{d} / a,
\end{array} .\right.
\end{aligned}
$$

It is easy to see that this time the numerator has the factor $\left(1-q^{(d-1) n}\right)\left(1-q^{(2-d) n}\right)$ and is therefore divisible by $\Phi_{n}(q)^{2}$, and again the denominator is coprime with $\Phi_{n}(q)$. This proves (4.1) for the first case.

Letting $a=1$ and $d=3$ in (4.1), we get

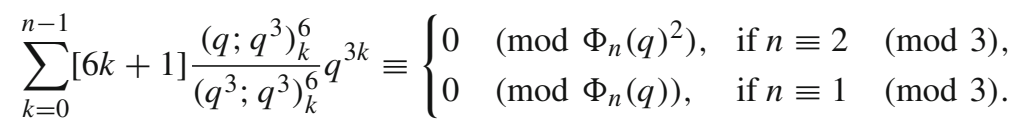

Similarly as in the proof of Theorem 2.1 , we can prove that

$$
\sum_{k=0}^{n-1}[6 k+1] \frac{\left(q ; q^{3}\right)_{k}^{6}}{\left(q^{3} ; q^{3}\right)_{k}^{6}} q^{3 k} \equiv 0 \quad(\bmod [n])
$$

This completes the proof of (2.6).

Likewise, taking $a \rightarrow 0$ in (4.1), we obtain

$$
\sum_{k=0}^{n-1}[2 d k+1] \frac{\left(q ; q^{d}\right)_{k}^{4}}{\left(q^{d} ; q^{d}\right)_{k}^{4}} q^{(d-2) k} \equiv\left\{\begin{array}{lll}
0 & \left(\bmod \Phi_{n}(q)^{2}\right), & \text { if } n \equiv-1 \quad(\bmod d) \\
0 & \left(\bmod \Phi_{n}(q)\right), & \text { otherwise }
\end{array}\right.
$$

This completes the proof of (2.7).

It appears that the following generalization with one more parameter $b$ is still true.

Conjecture 4.2 Let $n$ and $d$ be positive integers with $d \geq 3$ and $\operatorname{gcd}(n, d)=1$. Then

$$
\begin{aligned}
& \sum_{k=0}^{n-1}[2 d k+1] \frac{\left(a q, q / a, b q, q / b ; q^{d}\right)_{k}\left(q ; q^{d}\right)_{k}^{2}}{\left(a q^{d}, q^{d} / a, b q^{d}, q^{d} / b ; q^{d}\right)_{k}\left(q^{d} ; q^{d}\right)_{k}^{2}} q^{(2 d-3) k} \\
& \quad \equiv\left\{\begin{array}{lll}
0 & \left(\bmod [n] \Phi_{n}(q)\right), & \text { if } n \equiv-1 \quad(\bmod d), \\
0 & (\bmod [n]), & \text { otherwise. }
\end{array}\right.
\end{aligned}
$$




\section{More $q$-Congruences from Watson's Transformation}

Throughout this section, $m$ always stands for $n-1$ or $(n+1) / 2$. Note that the special case of [28, Thm. 4.9] with $r=-1, d=2$ and $a=1$ gives

$$
\begin{aligned}
& \sum_{k=0}^{m}(-1)^{k}[4 k-1] \frac{\left(q^{-1} ; q^{2}\right)_{k}^{3}}{\left(q^{2} ; q^{2}\right)_{k}^{3}} q^{k^{2}+2 k} \\
& \quad \equiv[n](-q)^{(n-3)(n+1) / 4} \quad\left(\bmod [n] \Phi_{n}(q)^{2}\right) \text { for odd } n>1 .
\end{aligned}
$$

In this section, we shall give some similar congruences.

Theorem 5.1 Let $n>1$ be an odd integer. Then

$$
\begin{aligned}
& \sum_{k=0}^{m}(-1)^{k}[4 k-1] \frac{\left(q^{-1} ; q^{2}\right)_{k}^{5}}{\left(q^{2} ; q^{2}\right)_{k}^{5}} q^{k^{2}+5 k} \\
& \quad \equiv[n](-q)^{(n+1)(n-3) / 4} \sum_{k=0}^{(n+1) / 2} \frac{\left(q^{-1} ; q^{2}\right)_{k}^{2}\left(q^{3} ; q^{2}\right)_{k}}{\left(q^{2} ; q^{2}\right)_{k}^{3}} q^{3 k} \quad\left(\bmod [n] \Phi_{n}(q)^{2}\right)
\end{aligned}
$$

Proof We first establish the following result:

$$
\begin{aligned}
& \sum_{k=0}^{(n+1) / 2}(-1)^{k}[4 k-1] \frac{\left(a q^{-1}, q^{-1} / a ; q^{2}\right)_{k}\left(q^{-1} ; q^{2}\right)_{k}^{3}}{\left(a q^{2}, q^{2} / a ; q^{2}\right)_{k}\left(q^{2} ; q^{2}\right)_{k}^{3}} q^{k^{2}+5 k} \equiv[n](-q)^{(n+1)(n-3) / 4} \\
& \quad \times \sum_{k=0}^{(n+1) / 2} \frac{\left(a q^{-1}, q^{-1} / a ; q^{2}\right)_{k}\left(q^{3} ; q^{2}\right)_{k}}{\left(q^{2} ; q^{2}\right)_{k}^{3}} q^{3 k}\left(\bmod \Phi_{n}(q)\left(1-a q^{n}\right)\left(a-q^{n}\right)\right) .
\end{aligned}
$$

For $a=q^{-n}$ or $a=q^{n}$, the left-hand side of (5.2) is equal to

$$
\begin{aligned}
& \sum_{k=0}^{(n+1) / 2}(-1)^{k}[4 k-1] \frac{\left(q^{-1-n}, q^{-1+n} ; q^{2}\right)_{k}\left(q^{-1} ; q^{2}\right)_{k}^{3}}{\left(q^{2-n}, q^{2+n} ; q^{2}\right)_{k}\left(q^{2} ; q^{2}\right)_{k}^{3}} q^{k^{2}+5 k} \\
& \quad=-q^{-1} \sum_{k=0}^{(n+1) / 2}(-1)^{k} \frac{\left(1-q^{4 k-1}\right)\left(q^{-1}, q^{-1}, q^{-1}, q^{-1-n}, q^{-1+n} ; q^{2}\right)_{k}}{\left(1-q^{-1}\right)\left(q^{2}, q^{2}, q^{2}, q^{2+n}, q^{2-n} ; q^{2}\right)_{k}} q^{k^{2}+5 k}
\end{aligned}
$$

By the limiting case of Watson's transformation formula (1.8), we can rewrite the right-hand side of (5.3) as

$$
\frac{-q^{-1}\left(q, q^{3} ; q^{2}\right)_{\infty}}{\left(q^{2+n}, q^{2-n} ; q^{2}\right)_{\infty}} \sum_{k=0}^{(n+1) / 2} \frac{\left(q^{-1-n}, q^{-1+n} ; q^{2}\right)_{k}\left(q^{3} ; q^{2}\right)_{k}}{\left(q^{2} ; q^{2}\right)_{k}^{3}} q^{3 k}
$$


It is easy to see that

$$
\frac{-q^{-1}\left(q, q^{3} ; q^{2}\right)_{\infty}}{\left(q^{2+n}, q^{2-n} ; q^{2}\right)_{\infty}}=\frac{-q^{-1}\left(q ; q^{2}\right)_{(n+1) / 2}}{\left(q^{2-n} ; q^{2}\right)_{(n+1) / 2}}=[n](-q)^{(n+1)(n-3) / 4} .
$$

This proves that the congruence (5.2) holds modulo $\left(1-a q^{n}\right)\left(a-q^{n}\right)$.

On the other hand, by Lemma 3.1, for $0 \leq k \leq(n+1) / 2$, we have

$$
\begin{aligned}
\frac{\left(a q^{-1} ; q^{2}\right)_{(n+1) / 2-k}}{\left(q^{2} / a ; q^{2}\right)_{(n+1) / 2-k}} & =\frac{\left(1-a q^{-1}\right)\left(a q ; q^{2}\right)_{(n-1) / 2-k}}{\left(1-q^{n+1-2 k} / a\right)\left(q^{2} / a ; q^{2}\right)_{(n-1) / 2-k}} \\
& \equiv(-a)^{(n-1) / 2-2 k} \frac{\left(1-a q^{-1}\right)\left(a q ; q^{2}\right)_{k}}{\left(1-q^{1-2 k} / a\right)\left(q^{2} / a ; q^{2}\right)_{k}} q^{(n-1)^{2} / 4+k} \\
& =(-a)^{(n+1) / 2-2 k} \frac{\left(a q^{-1} ; q^{2}\right)_{k}}{\left(q^{2} / a ; q^{2}\right)_{k}} q^{(n-1)^{2} / 4+3 k-1} \quad\left(\bmod \Phi_{n}(q)\right) .
\end{aligned}
$$

It follows that the $k$ th and $((n+1) / 2-k)$ th terms on the left-hand side of (5.2) cancel each other modulo $\Phi_{n}(q)$. When the respective sum has an odd number of factors, the central term will remain. This happens when $n=4 l-1$ for some positive integer $l$, and in this case the central term has index $k=l$ and one directly sees that $[4 k-1]=[n]$ is a factor of the summand. In total, this proves that the congruence (5.2) also holds modulo $\Phi_{n}(q)$. Since the polynomials $\Phi_{n}(q), 1-a q^{n}$ and $a-q^{n}$ are coprime with one another, the proof of (5.2) is complete.

Letting $a \rightarrow 1$ in (5.2), one sees that the congruence (5.1) holds modulo $\Phi_{n}(q)^{3}$ by noticing that $\left(q^{-1} ; q^{2}\right)_{5}^{k} /\left(q^{2} ; q^{2}\right)_{k}^{5} \equiv 0\left(\bmod \Phi_{n}(q)^{3}\right)$ for $(n+1) / 2<k \leq n-1$. Along the same lines of the proof of Theorem 2.1, we can prove that

$$
\sum_{k=0}^{m}(-1)^{k}[4 k-1] \frac{\left(q^{-1} ; q^{2}\right)_{k}^{5}}{\left(q^{2} ; q^{2}\right)_{k}^{5}} q^{k^{2}+5 k} \equiv 0 \quad(\bmod [n])
$$

i.e., the congruence (5.1) holds modulo $[n]$. Since $\operatorname{lcm}\left(\Phi_{n}(q)^{3},[n]\right)=[n] \Phi_{n}(q)^{2}$, the proof of the theorem is complete.

Corollary 5.2 We have

$$
\sum_{k=0}^{(p+1) / 2}(-1)^{k}(4 k-1) \frac{\left(-\frac{1}{2}\right)_{k}^{5}}{k !^{5}} \equiv(-1)^{(p+1) / 2} p \sum_{k=0}^{(p+1) / 2} \frac{\left(-\frac{1}{2}\right)_{k}^{2}\left(\frac{3}{2}\right)_{k}}{k !^{3}}\left(\bmod p^{3}\right)
$$

Theorem 5.3 Let $n>1$ be an odd integer. Then

$$
\sum_{k=0}^{m}[4 k-1] \frac{\left(q^{-1} ; q^{2}\right)_{k}^{4}}{\left(q^{2} ; q^{2}\right)_{k}^{4}} q^{4 k} \equiv 0 \quad\left(\bmod [n] \Phi_{n}(q)^{2}\right)
$$


Proof We first establish the following congruence:

$$
\sum_{k=0}^{m}[4 k-1] \frac{\left(a q^{-1}, q^{-1} / a ; q^{2}\right)_{k}\left(q^{-1} ; q^{2}\right)_{k}^{2}}{\left(a q^{2}, q^{2} / a ; q^{2}\right)_{k}\left(q^{2} ; q^{2}\right)_{k}^{2}} q^{4 k} \equiv 0 \quad\left(\bmod \Phi_{n}(q)\left(1-a q^{n}\right)\left(a-q^{n}\right)\right) .
$$

Letting $q \mapsto q^{2}$ and $c \rightarrow 0$ followed by $a=b=q^{-1}, d=q^{-1-n}$ and $e=q^{-1+n}$ in the limiting case of Watson's transformation formula (1.8), we obtain

$$
\begin{aligned}
& \sum_{k=0}^{m}[4 k-1] \frac{\left(q^{-1-n}, q^{-1+n} ; q^{2}\right)_{k}\left(q^{-1} ; q^{2}\right)_{k}^{2}}{\left(q^{2-n}, q^{2+n} ; q^{2}\right)_{k}\left(q^{2} ; q^{2}\right)_{k}^{2}} q^{4 k} \\
& \quad=\frac{-q^{-1}\left(q, q^{3} ; q^{2}\right)_{\infty}}{\left(q^{2+n}, q^{2-n} ; q^{2}\right)_{\infty}} \sum_{k=0}^{(n+1) / 2} \frac{\left(q^{-1-n}, q^{-1+n} ; q^{2}\right)_{k}}{\left(q^{2} ; q^{2}\right)_{k}^{2}} q^{4 k}
\end{aligned}
$$

By the $q$-Chu-Vandermonde summation formula [13, Appendix (II.6)], for odd $n>1$, we have

$$
\sum_{k=0}^{m} \frac{\left(q^{-1-n}, q^{-1+n} ; q^{2}\right)_{k}}{\left(q^{2} ; q^{2}\right)_{k}^{2}} q^{2 k}=\frac{\left(q^{3-n} ; q^{2}\right)_{(n+1) / 2}}{\left(q^{2} ; q^{2}\right)_{(n+1) / 2}} q^{\left(n^{2}-1\right) / 2}=0 .
$$

Letting $q \mapsto q^{-1}$ in the above equality, we see that the summation on the righthand side of (5.7) is equal to 0 . This proves that the congruence (5.6) holds modulo $\left(1-a q^{n}\right)\left(a-q^{n}\right)$.

On the other hand, similarly as before, by (5.4) one sees that the sum of the $k$ th and $((n+1) / 2-k)$ th terms on the left-hand side of (5.6) are congruent to 0 modulo $\Phi_{n}(q)$ (and also, when the respective sum has an odd number of factors, i.e., when $n=4 l-1$ for some positive integer $l$, then the remaining central term has index $k=l$ and one directly sees that $[4 k-1]=[n]$ is a factor of the summand). This thus proves that the congruence (5.6) is also true modulo $\Phi_{n}(q)$. This completes the proof of (5.6).

Let $c_{q}(k)$ denote the $k$ th term on the left-hand side of (5.6). In the same vein as in the proof of Theorem 2.1, we can further prove that

$$
\sum_{k=0}^{n-1} c_{q}(k)=\sum_{k=0}^{(n+1) / 2} c_{q}(k) \equiv 0 \quad(\bmod [n])
$$

Thus, we have proved that

$$
\sum_{k=0}^{m}[4 k-1] \frac{\left(a q^{-1}, q^{-1} / a ; q^{2}\right)_{k}\left(q^{-1} ; q^{2}\right)_{k}^{2}}{\left(a q^{2}, q^{2} / a ; q^{2}\right)_{k}\left(q^{2} ; q^{2}\right)_{k}^{2}} q^{4 k} \equiv 0 \quad\left(\bmod [n]\left(1-a q^{n}\right)\left(a-q^{n}\right)\right) .
$$


The parts of the denominators in (5.10) which contain the parameter $a$ are the factors of $\left(a q^{2}, q^{2} / a ; q^{2}\right)_{(n+1) / 2}$ or $\left(a q^{2}, q^{2} / a ; q^{2}\right)_{n-1}$. Their limits as $a \rightarrow 1$ are relatively prime to $\Phi_{n}(q)$. On the other hand, the limit of $\left(1-a q^{n}\right)\left(a-q^{n}\right)$ as $a \rightarrow 1$ has the factor $\Phi_{n}(q)^{2}$. Therefore, the limiting case $a \rightarrow 1$ of the congruence (5.10) reduces to (5.5) modulo $\Phi_{n}(q)^{3}$. But the congruences (5.9) are still true when $a=1$ which implies that the congruence (5.5) holds modulo $[n]$. This completes the proof of the theorem.

It appears that the congruence conditions stated in Theorem 5.3 and its extension in (5.6) can be strengthened:

Conjecture 5.4 Let $n>1$ be an odd integer. Then

$$
\begin{aligned}
& \sum_{k=0}^{m}[4 k-1] \frac{\left(a q^{-1} ; q^{2}\right)_{k}\left(q^{-1} / a ; q^{2}\right)_{k}\left(q^{-1} ; q^{2}\right)_{k}^{2}}{\left(a q^{2} ; q^{2}\right)_{k}\left(q^{2} / a ; q^{2}\right)_{k}\left(q^{2} ; q^{2}\right)_{k}^{2}} q^{4 k} \equiv 0 \\
& \left(\bmod [n]^{2}\left(1-a q^{n}\right)\left(a-q^{n}\right)\right),
\end{aligned}
$$

and

$$
\sum_{k=0}^{m}[4 k-1] \frac{\left(q^{-1} ; q^{2}\right)_{k}^{4}}{\left(q^{2} ; q^{2}\right)_{k}^{4}} q^{4 k} \equiv 0 \quad\left(\bmod [n]^{4}\right)
$$

Theorem 5.5 Let $n>3$ be an odd integer. Then

$$
\sum_{k=0}^{(n+1) / 2}[4 k+1] \frac{\left(q^{-1} ; q^{2}\right)_{k}^{2}\left(q ; q^{2}\right)_{k}^{2}}{\left(q^{4} ; q^{2}\right)_{k}^{2}\left(q^{2} ; q^{2}\right)_{k}^{2}} q^{4 k} \equiv 0 \quad\left(\bmod [n] \Phi_{n}(q)^{2}\right)
$$

Proof The proof is similar to that of Theorem 5.3. We first establish

$$
\sum_{k=0}^{(n+1) / 2}[4 k+1] \frac{\left(a q^{-1}, q^{-1} / a ; q^{2}\right)_{k}\left(q ; q^{2}\right)_{k}^{2}}{\left(a q^{4}, q^{4} / a ; q^{2}\right)_{k}\left(q^{2} ; q^{2}\right)_{k}^{2}} q^{4 k} \equiv 0 \quad\left(\bmod \Phi_{n}(q)\left(1-a q^{n}\right)\left(a-q^{n}\right)\right)
$$

for odd $n>1$. Letting $q \mapsto q^{2}$ and $c \rightarrow 0$ followed by $a=b=q, d=q^{-1-n}$, and $e=q^{-1+n}$ in (1.8), we obtain

$$
\begin{aligned}
& \sum_{k=0}^{(n+1) / 2}[4 k+1] \frac{\left(q^{-1-n}, q^{-1+n} ; q^{2}\right)_{k}\left(q ; q^{2}\right)_{k}^{2}}{\left(q^{4-n}, q^{4+n} ; q^{2}\right)_{k}\left(q^{2} ; q^{2}\right)_{k}^{2}} q^{4 k} \\
& =\frac{\left(q^{3}, q^{5} ; q^{2}\right)_{\infty}}{\left(q^{4+n}, q^{4-n} ; q^{2}\right)_{\infty}} \sum_{k=0}^{(n+1) / 2} \frac{\left(q^{-1-n}, q^{-1+n} ; q^{2}\right)_{k}}{\left(q^{2} ; q^{2}\right)_{k}^{2}} q^{4 k}
\end{aligned}
$$


As we have already mentioned in the proof of Theorem 5.3, the summation on the right-hand side of (5.13) is equal to 0 by the $q \mapsto q^{-1}$ case of (5.8). Thus, we have proved that the congruence (5.12) holds modulo $\left(1-a q^{n}\right)\left(a-q^{n}\right)$.

On the other hand, similarly as before, by (5.4) one sees that the sum of the $k$ th and $((n-1) / 2-k)$ th terms on the left-hand side of (5.12) are congruent to 0 modulo $\Phi_{n}(q)$ for $0 \leq k \leq(n-1) / 2$. Moreover, the summand for $k=(n+1) / 2$ on the right-hand side of (5.13) is clearly congruent to 0 modulo $\Phi_{n}(q)$ because of the factor $\left(q ; q^{2}\right)_{(n+1) / 2}$ in the numerator. This proves that the congruence $(5.12)$ is also true modulo $\Phi_{n}(q)$. The proof of (5.12) is completed.

For $n>3$, we have $(n+3) / 2<n$ and so the denominator of the left-hand side of (5.12) is relatively prime to $\Phi_{n}(q)$ when taking the limit as $a \rightarrow 1$. Therefore, the congruence (5.11) holds modulo $\Phi_{n}(q)^{3}$ for $n>3$ by taking $a \rightarrow 1$ in (5.12). On the other hand, it is also easy to see that the congruence (5.11) holds modulo $\Phi_{3}(q)$ for $n=3$. Let $c_{q}(k)$ denote the $k$ th term on the left-hand side of (5.12). Similarly to the proof of Theorem 2.1, we can further prove that

$$
\sum_{k=0}^{n-2} c_{q}(k)=\sum_{k=0}^{(n+1) / 2} c_{q}(k) \equiv 0 \quad(\bmod [n])
$$

This proves (5.11).

We conjecture that the following generalization of (5.12) and Theorem 5.5 is still true.

Conjecture 5.6 Let $n>3$ be an odd integer. Then

$$
\begin{aligned}
& \sum_{k=0}^{(n+1) / 2}[4 k+1] \frac{\left(a q^{-1} ; q^{2}\right)_{k}\left(q^{-1} / a ; q^{2}\right)_{k}\left(q ; q^{2}\right)_{k}^{2}}{\left(a q^{4} ; q^{2}\right)_{k}\left(q^{4} / a ; q^{2}\right)_{k}\left(q^{2} ; q^{2}\right)_{k}^{2}} q^{4 k} \equiv 0 \\
& \quad\left(\bmod [n] \Phi_{n}(q)\left(1-a q^{n}\right)\left(a-q^{n}\right)\right)
\end{aligned}
$$

in particular,

$$
\sum_{k=0}^{(n+1) / 2}[4 k+1] \frac{\left(q^{-1} ; q^{2}\right)_{k}^{2}\left(q ; q^{2}\right)_{k}^{2}}{\left(q^{4} ; q^{2}\right)_{k}^{2}\left(q^{2} ; q^{2}\right)_{k}^{2}} q^{4 k} \equiv 0 \quad\left(\bmod [n] \Phi_{n}(q)^{3}\right)
$$

Analogously, letting $q \mapsto q^{2}$ and $c \rightarrow 0$ followed by $a=q, b=q^{-1}, d=q^{-1-n}$, and $e=q^{-1+n}$ in (1.8), we can prove the following result:

$$
\begin{gathered}
\sum_{k=0}^{(n+1) / 2}[4 k+1] \frac{\left(a q^{-1} ; q^{2}\right)_{k}\left(q^{-1} / a ; q^{2}\right)_{k}\left(q^{-1}, q ; q^{2}\right)_{k}}{\left(a q^{4} ; q^{2}\right)_{k}\left(q^{4} / a ; q^{2}\right)_{k}\left(q^{4}, q^{2} ; q^{2}\right)_{k}} q^{6 k} \equiv 0 \\
\left(\bmod \Phi_{n}(q)\left(1-a q^{n}\right)\left(a-q^{n}\right)\right) .
\end{gathered}
$$

We label the limiting case $a \rightarrow 1$ as the following theorem. 
Theorem 5.7 Let $n>3$ be an odd integer. Then

$$
\sum_{k=0}^{(n+1) / 2}[4 k+1] \frac{\left(q^{-1} ; q^{2}\right)_{k}^{3}\left(q ; q^{2}\right)_{k}}{\left(q^{4} ; q^{2}\right)_{k}^{3}\left(q^{2} ; q^{2}\right)_{k}} q^{6 k} \equiv 0 \quad\left(\bmod \Phi_{n}(q)^{3}\right) .
$$

Moreover, if $\operatorname{gcd}(n, 3)=1$, then the above congruence holds modulo $[n] \Phi_{n}(q)^{2}$.

It seems that the following generalization of (5.14) and (5.15) still holds.

Conjecture 5.8 Let $n>3$ be a positive odd integer. Then

$$
\begin{aligned}
& \sum_{k=0}^{(n+1) / 2}[4 k+1] \frac{\left(a q^{-1} ; q^{2}\right)_{k}\left(q^{-1} / a ; q^{2}\right)_{k}\left(q^{-1}, q ; q^{2}\right)_{k}}{\left(a q^{4} ; q^{2}\right)_{k}\left(q^{4} / a ; q^{2}\right)_{k}\left(q^{4}, q^{2} ; q^{2}\right)_{k}} q^{6 k} \equiv 0 \\
& \quad\left(\bmod \Phi_{n}^{2}(q)\left(1-a q^{n}\right)\left(a-q^{n}\right)\right)
\end{aligned}
$$

in particular,

$$
\sum_{k=0}^{(n+1) / 2}[4 k+1] \frac{\left(q^{-1} ; q^{2}\right)_{k}^{3}\left(q ; q^{2}\right)_{k}}{\left(q^{4} ; q^{2}\right)_{k}^{3}\left(q^{2} ; q^{2}\right)_{k}} q^{6 k} \equiv 0 \quad\left(\bmod \Phi_{n}(q)^{4}\right)
$$

We also have the following similar result.

Theorem 5.9 Let $n>1$ be a positive odd integer. Then

$$
\sum_{k=0}^{(n-1) / 2}[4 k+1] \frac{\left(q^{-1} ; q^{2}\right)_{k}\left(q ; q^{2}\right)_{k}^{3}}{\left(q^{4} ; q^{2}\right)_{k}\left(q^{2} ; q^{2}\right)_{k}^{3}} q^{2 k} \equiv 0 \quad\left(\bmod [n]^{3}\right) .
$$

Proof It is easy to see by induction on $N$ that

$$
\sum_{k=0}^{N}[4 k+1] \frac{\left(q^{-1} ; q^{2}\right)_{k}\left(q ; q^{2}\right)_{k}^{3}}{\left(q^{4} ; q^{2}\right)_{k}\left(q^{2} ; q^{2}\right)_{k}^{3}} q^{2 k}=\frac{\left(q ; q^{2}\right)_{N}\left(q ; q^{2}\right)_{N+1}^{3}}{(1-q)^{3}\left(q^{4} ; q^{2}\right)_{N}\left(q^{2} ; q^{2}\right)_{N}^{3}} .
$$

Putting $N=(n-1) / 2$ in the above identity and using (1.6), we get

$$
\begin{aligned}
\sum_{k=0}^{(n-1) / 2}[4 k+1] \frac{\left(q^{-1} ; q^{2}\right)_{k}\left(q ; q^{2}\right)_{k}^{3}}{\left(q^{4} ; q^{2}\right)_{k}\left(q^{2} ; q^{2}\right)_{k}^{3} q^{2 k}} & =\frac{[n]^{3}\left(q ; q^{2}\right)_{(n-1) / 2}^{4}}{\left(q^{4} ; q^{2}\right)_{(n-1) / 2}\left(q^{2} ; q^{2}\right)_{(n-1) / 2}^{3}} \\
& =\frac{[n]^{3}(1+q)}{[n+1](-q ; q)_{(n-1) / 2}^{8}}\left[\begin{array}{c}
n-1 \\
(n-1) / 2
\end{array}\right]^{4}
\end{aligned}
$$

The proof then follows from the fact that $\operatorname{gcd}([n],[n+1])=1$ and $\operatorname{gcd}\left([n],(-q ; q)_{N}\right)=$ 1 (for odd $n$ ). 
Let $n=p$ and $q=1$ in (5.17). Using Fermat's little theorem, we immediately obtain the following conclusion.

Corollary 5.10 We have

$$
\sum_{k=0}^{(p-1) / 2}(4 k+1) \frac{\left(-\frac{1}{2}\right)_{k}\left(\frac{1}{2}\right)_{k}^{3}}{(k+1) ! k !^{3}} \equiv 2 p^{3} \quad\left(\bmod p^{4}\right)
$$

We end this section with the following conjecture, which is similar to Conjecture 4.2. As in the proof of Theorem 4.1, we can confirm it for $b=1$.

Conjecture 5.11 Let $n$ and $d$ be positive integers with $d \geq 3$ and $\operatorname{gcd}(n, d)=1$. Then

$$
\begin{aligned}
& \sum_{k=0}^{n-1}[2 d k-1] \frac{\left(a q^{-1}, q^{-1} / a, b q^{-1}, q^{-1} / b ; q^{d}\right)_{k}\left(q^{-1} ; q^{d}\right)_{k}^{2}}{\left(a q^{d}, q^{d} / a, b q^{d}, q^{d} / b ; q^{d}\right)_{k}\left(q^{d} ; q^{d}\right)_{k}^{2}} q^{(2 d+3) k} \\
& \quad \equiv\left\{\begin{array}{lll}
0 & \left(\bmod [n] \Phi_{n}(q)\right), & \text { if } n \equiv 1 \quad(\bmod d), \\
0 & (\bmod [n]), & \text { otherwise. }
\end{array}\right.
\end{aligned}
$$

\section{Proof of a Three-Parametric $q$-Congruence from a Quadratic Transformation of Rahman}

In this section, we confirm a three-parametric $q$-congruence conjecture of the first author and Zudilin [28, Conj. 4.6].

Theorem 6.1 Let $n$ be a positive odd integer. Then, modulo $[n]\left(1-a q^{n}\right)\left(a-q^{n}\right)$,

$$
\begin{aligned}
& \sum_{k=0}^{M}[3 k+1] \frac{\left(a q, q / a, q ; q^{2}\right)_{k}(q / b, q / c, b c ; q)_{k}}{(a q, q / a, q ; q)_{k}\left(b q^{2}, c q^{2}, q^{3} / b c ; q^{2}\right)_{k}} q^{k} \\
& \quad \equiv \frac{\left(b c q, q^{2} / b, q^{2} / c ; q^{2}\right)_{(n-1) / 2}}{\left(q^{3} / b c, b q^{2}, c q^{2} ; q^{2}\right)_{(n-1) / 2}}[n]
\end{aligned}
$$

where $M=n-1$ or $(n-1) / 2$.

The congruence (6.1) modulo $\left(1-a q^{n}\right)\left(a-q^{n}\right)$ has already been proved by the first author and Zudilin [28, Thm. 4.7]. Moreover, the congruence (6.1) with $c=1$ was established in [28, Thm. 4.8]. Therefore, it remains to prove that (6.1) holds modulo $[n]$; i.e.,

$$
\sum_{k=0}^{M}[3 k+1] \frac{\left(a q, q / a, q ; q^{2}\right)_{k}(q / b, q / c, b c ; q)_{k}}{(a q, q / a, q ; q)_{k}\left(b q^{2}, c q^{2}, q^{3} / b c ; q^{2}\right)_{k}} q^{k} \equiv 0 \quad(\bmod [n])
$$


Proof We need to use a quadratic transformation formula of Rahman [47] (see also [13, Eq. (3.8.13)]):

$$
\begin{aligned}
& \sum_{k=0}^{\infty} \frac{\left(1-a q^{3 k}\right)\left(a, d, a q / d ; q^{2}\right)_{k}(b, c, a q / b c ; q)_{k}}{(1-a)(a q / d, d, q ; q)_{k}\left(a q^{2} / b, a q^{2} / c, b c q ; q^{2}\right)_{k}} q^{k} \\
& \quad=\frac{\left(a q^{2}, b q, c q, a q^{2} / b c ; q^{2}\right)_{\infty}}{\left(q, a q^{2} / b, a q^{2} / c, b c q ; q^{2}\right)_{\infty}} 3 \phi_{2}\left[\begin{array}{c}
b, c, a q / b c \\
d q, a q^{2} / d
\end{array} ; q^{2}, q^{2}\right]
\end{aligned}
$$

provided $d$ or $a q / d$ is not of the form $q^{-2 n}, n$ a non-negative integer. It is clear that (6.2) is true for $n=1$. We now suppose that $n>1$. Let $a=q^{1-n}$ in (6.3) and then we further set $d=a q$ and replace $b$ and $c$ with $q / b$ and $q / c$, respectively. Then the left-hand side of (6.3) terminates at $k=(n-1) / 2$, and the right-side of (6.3) vanishes because the numerator contains the factor $\left(q^{3-n} ; q^{2}\right)_{\infty}$. Namely, we have

$$
\sum_{k=0}^{(n-1) / 2} \frac{1-q^{3 k+1-n}}{1-q^{1-n}} \frac{\left(a q, q^{1-n} / a, q^{1-n} ; q^{2}\right)_{k}\left(q / b, q / c, b c q^{-n} ; q\right)_{k}}{\left(a q, q^{1-n} / a, q ; q\right)_{k}\left(b q^{2-n}, c q^{2-n}, q^{3} / b c ; q^{2}\right)_{k}} q^{k}=0
$$

Since $q^{n} \equiv 1\left(\bmod \Phi_{n}(q)\right)$, we immediately get

$$
\sum_{k=0}^{(n-1) / 2}[3 k+1] \frac{\left(a q, q / a, q ; q^{2}\right)_{k}(q / b, q / c, b c ; q)_{k}}{(a q, q / a, q ; q)_{k}\left(b q^{2}, c q^{2}, q^{3} / b c ; q^{2}\right)_{k}} q^{k} \equiv 0 \quad\left(\bmod \Phi_{n}(q)\right)
$$

Finally, the proof of (6.2) is completely analogous to that of Theorem 2.1 (more precisely, to the proofs of (3.7a) and (3.7b)).

Letting $a, b \rightarrow 1$ and $c \rightarrow 0$ in (6.1), we obtain

$$
\sum_{k=0}^{M}[3 k+1] \frac{\left(q ; q^{2}\right)_{k}^{3} q^{-\left(\begin{array}{c}
k+1 \\
2
\end{array}\right)}}{(q ; q)_{k}^{2}\left(q^{2} ; q^{2}\right)_{k}} \equiv q^{(1-n) / 2}[n] \quad\left(\bmod [n] \Phi_{n}(q)^{2}\right)
$$

(see also [21]), while letting $a, b \rightarrow 1$ and $c \rightarrow-1$ in (6.1), we get

$$
\sum_{k=0}^{M}[3 k+1] \frac{\left(q ; q^{2}\right)_{k}^{3}(-1 ; q)_{k} q^{k}}{(q ; q)_{k}^{3}\left(-q^{2},-q^{3} ; q^{2}\right)_{k}} \equiv \frac{1+q}{1+q^{n}}[n] \quad\left(\bmod [n] \Phi_{n}(q)^{2}\right) .
$$

It is easy to see that both (6.4) and (6.5) are $q$-analogues of the following supercongruence:

$$
\sum_{k=0}^{M}(3 k+1) \frac{\left(\frac{1}{2}\right)_{k}^{3}}{k !^{3}} 2^{2 k} \equiv p \quad\left(\bmod p^{3}\right)
$$


where $M=p-1$ or $M=(p-1) / 2$. The congruence (6.6) with $M=(p-1) / 2$ was first proved by Guillera and Zudilin [15] using the WZ (Wilf-Zeilberger) method. For $M=p-1, \mathrm{Hu}[30]$ proved an even stronger congruence, namely

$$
\sum_{k=0}^{p-1}(3 k+1) \frac{\left(\frac{1}{2}\right)_{k}^{3}}{k !^{3}} 2^{2 k} \equiv p \quad\left(\bmod p^{4}\right)
$$

Also the $M=(p-1) / 2$ case of (6.6) can be extended to a congruence modulo $p^{4}$, namely

$$
\sum_{k=0}^{(p-1) / 2}(3 k+1) \frac{\left(\frac{1}{2}\right)_{k}^{3}}{k !^{3}} 2^{2 k} \equiv p+(-1)^{(p-1) / 2} 2 p^{3} E_{p-3} \quad\left(\bmod p^{4}\right)
$$

where $E_{p-3}$ is the $(p-3)$ th Euler number, which was conjectured by Sun [51, Conj. 5.1(ii)] and recently proved by Mao and Zhang [42].

Here we would like to propose a supercongruence similar to (6.6).

Conjecture 6.2 We have

$$
\sum_{k=0}^{(p+1) / 2}(3 k-1) \frac{\left(-\frac{1}{2}\right)_{k}^{2}\left(\frac{1}{2}\right) k}{k !^{3}} 2^{2 k} \equiv p \quad\left(\bmod p^{3}\right)
$$

Unfortunately, we were not able to find any $q$-analogue of (6.9), even for the simple case modulo $p$.

Moreover, letting $a \rightarrow 1, b \rightarrow-1$, and $c \rightarrow 0$ in (6.1), we get

$$
\sum_{k=0}^{M}(-1)^{k}[3 k+1] \frac{\left(q ; q^{2}\right)_{k}^{3}(-q ; q)_{k} q^{-\left(\begin{array}{c}
k+1 \\
2
\end{array}\right)}}{(q ; q)_{k}^{3}\left(-q^{2} ; q^{2}\right)_{k}} \equiv[n](-q)^{(1-n) / 2} \quad\left(\bmod [n] \Phi_{n}(q)^{2}\right),
$$

while letting $a \rightarrow 1$ and $b, c \rightarrow 0$ in (6.1), we arrive at

$$
\sum_{k=0}^{M}(-1)^{k}[3 k+1] \frac{\left(q ; q^{2}\right)_{k}^{3}}{(q ; q)_{k}^{3}} \equiv[n](-q)^{(n-1)^{2} / 4} \quad\left(\bmod [n] \Phi_{n}(q)^{2}\right)
$$

It is worth mentioning that both (6.10) and (6.11) are $q$-analogues of the following supercongruence due to Guillera and Zudilin [15]:

$$
\sum_{k=0}^{(p-1) / 2}(-1)^{k}(3 k+1) \frac{\left(\frac{1}{2}\right)_{k}^{3}}{k !^{3}} 2^{3 k} \equiv p(-1)^{(p-1) / 2} \quad\left(\bmod p^{3}\right)
$$

The congruence (6.11) with $M=n-1$ was first established by the first author [21] using the $q$-WZ method. The congruence (6.10) is new. 
Sun [51, Conj. 5.1(ii)] conjectured that

$$
\sum_{k=0}^{p-1}(-1)^{k}(3 k+1) \frac{\left(\frac{1}{2}\right)_{k}^{3}}{k !^{3}} 2^{3 k} \equiv p(-1)^{(p-1) / 2}+p^{3} E_{p-3} \quad\left(\bmod p^{4}\right)
$$

(where $E_{p-3}$ is again the $(p-3)$ th Euler number), for any prime $p>3$, which was confirmed by Chen, Xie and He [8].

Motivated by Conjecture 6.2, (6.8) and (6.13), we would like to raise the following problems.

Problem 6.3 Is there a " $3 k-1$ version" of the supercongruence (6.12)?

Problem 6.4 Are there any q-analogues of hypergeometric supercongruences involving Euler numbers as in (6.8) or (6.13)?

\section{Some $q$-Congruences from a Cubic Transformation of Gasper and Rahman}

Gasper and Rahman [12] (see also [13, Eq. (3.8.18)]) obtained the following cubic transformation:

$$
\begin{aligned}
\sum_{k=0}^{\infty} & \frac{1-a c q^{4 k}}{1-a c} \frac{(a, q / a ; q)_{k}(a c ; q)_{2 k}\left(d, a c q / d ; q^{3}\right)_{k}}{\left(c q^{3}, a^{2} c q^{2} ; q^{3}\right)_{k}(q ; q)_{2 k}(a c q / d, d ; q)_{k}} q^{k} \\
= & \frac{\left(a c q^{2}, a c q^{3}, d / a c, d q / a c, a d q, a q, q^{2} / a, d q^{2} / a ; q^{3}\right)_{\infty}}{\left(q, q^{2}, d q, d q^{2}, a^{2} c q^{2}, c q^{3}, d q / a^{2} c, d / c ; q^{3}\right)_{\infty}} \\
& +\frac{d(a, q / a, a c q ; q)_{\infty}\left(q^{3}, d, a c q / d, d^{2} q^{2} / a c ; q^{3}\right)_{\infty}}{a c(q, d, a c q / d ; q)_{\infty}\left(c q^{3}, a^{2} c q^{2}, d / c, d q / a^{2} c ; q^{3}\right)_{\infty}} \\
& \times{ }_{2} \phi_{1}\left[\begin{array}{c}
d / c, d q / a^{2} c \\
d^{2} q^{2} / a c
\end{array} ; q^{3}, q^{3}\right] .
\end{aligned}
$$

This transformation for $d=0$ becomes a summation formula and has been used by the first author and Zudilin [28] to prove the following $q$-congruence: modulo $[n]\left(1-a q^{n}\right)\left(a-q^{n}\right)$,

$$
\sum_{k=0}^{M}[8 k+1] \frac{\left(a q, q / a ; q^{2}\right)_{k}\left(q ; q^{2}\right)_{2 k}}{\left(q^{2} ; q^{2}\right)_{2 k}\left(a q^{6}, q^{6} / a ; q^{6}\right)_{k}} q^{2 k^{2}} \equiv q^{-(n-1) / 2}[n]\left(\frac{-3}{n}\right)
$$

where $M=n-1$ or $(n-1) / 2,(\vdots)$ is the Jacobi-Kronecker symbol, and $\operatorname{gcd}(n, 6)=1$. In this section, we shall deduce some $q$-congruences from (7.1) with $d \neq 0$.

Theorem 7.1 Let $n>1$ be an integer coprime with 6 . Then 


$$
\begin{aligned}
& \sum_{k=0}^{(n-1) / 2}[8 k+1] \frac{\left(a q, q / a ; q^{2}\right)_{k}\left(q ; q^{2}\right)_{2 k}\left(q, q^{2} ; q^{6}\right)_{k}}{\left(q^{2} ; q^{2}\right)_{2 k}\left(a q^{6}, q^{6} / a ; q^{6}\right)_{k}(q ; q)_{2 k}} q^{2 k}
\end{aligned}
$$

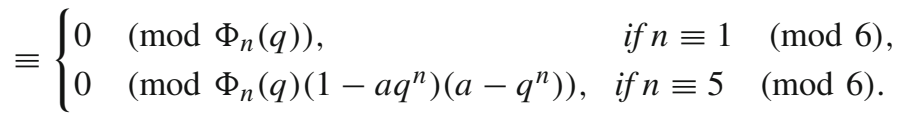

Proof We specialize (7.1) by taking $q \mapsto q^{2}$, then $c=q^{1-n} / a, d=q$, and replace $a$ by $a q$. The right-hand side of the resulting identity vanishes, because the numerator of the first fraction contains the factors $\left(q^{5-n} ; q^{6}\right)_{\infty}$ and $\left(q^{7-n} ; q^{6}\right)_{\infty}$, and the numerator of the second fraction has the factor $\left(q^{3-n} ; q^{2}\right)_{\infty}$. At the same time, the left-hand side terminates at $k=(n-1) / 2$ (in fact, much earlier, at $k=\lfloor n / 4\rfloor$ ), for the summand involves the term $\left(q^{1-n} ; q^{2}\right)_{2 k}$. This proves that

$$
\sum_{k=0}^{(n-1) / 2} \frac{1-q^{1-n+8 k}}{1-q^{1-n}} \frac{\left(a q, q / a ; q^{2}\right)_{k}\left(q^{1-n} ; q^{2}\right)_{2 k}\left(q, q^{2-n} ; q^{6}\right)_{k}}{\left(q^{2} ; q^{2}\right)_{2 k}\left(a q^{6-n}, q^{6-n} / a ; q^{6}\right)_{k}\left(q^{2-n} ; q^{2}\right)_{k}\left(q ; q^{2}\right)_{k}} q^{2 k}=0 .
$$

Since $q^{n} \equiv 1\left(\bmod \Phi_{n}(q)\right)$, we deduce that the congruence (7.3) holds modulo $\Phi_{n}(q)$.

We now assume that $n \equiv 5(\bmod 6)$. It remains to show that

$$
\sum_{k=0}^{(n-1) / 2}[8 k+1] \frac{\left(a q, q / a ; q^{2}\right)_{k}\left(q ; q^{2}\right)_{2 k}\left(q, q^{2} ; q^{6}\right)_{k}}{\left(q^{2} ; q^{2}\right)_{2 k}\left(a q^{6}, q^{6} / a ; q^{6}\right)_{k}(q ; q)_{2 k}} q^{2 k} \equiv 0 \quad\left(\bmod \left(1-a q^{n}\right)\left(a-q^{n}\right)\right)
$$

or equivalently,

$$
\sum_{k=0}^{(n-1) / 2}[8 k+1] \frac{\left(q^{1-n}, q^{1+n} ; q^{2}\right)_{k}\left(q ; q^{2}\right)_{2 k}\left(q, q^{2} ; q^{6}\right)_{k}}{\left(q^{2} ; q^{2}\right)_{2 k}\left(q^{6-n}, q^{6+n} ; q^{6}\right)_{k}(q ; q)_{2 k}} q^{2 k}=0
$$

This identity again follows straightforwardly from (7.1) by setting $q \mapsto q^{2}, a=q^{1-n}$, $c=q^{n}$, and $d=q$. In fact, the left-hand side of the resulting identity terminates at $k=(n-1) / 2$ and is therefore just the left-hand side of (7.4). On the other hand, the right-hand side of the resulting identity vanishes because the first fraction is equal to

$$
\frac{\left(q^{5}, q^{7}, 1, q^{2}, q^{4-n}, q^{3-n}, q^{3+n}, q^{4+n} ; q^{6}\right)_{\infty}}{\left(q^{2}, q^{4}, q^{3}, q^{5}, q^{6-n}, q^{6+n}, q^{1+n}, q^{1-n} ; q^{6}\right)_{\infty}}=0
$$

and the second fraction is equal to

$$
\frac{\left(q^{1-n}, q^{1+n}, q^{3} ; q^{2}\right)_{\infty}\left(q^{6}, q, q^{2}, q^{5} ; q^{6}\right)_{\infty}}{\left(q^{2}, q, q^{2} ; q^{2}\right)_{\infty}\left(q^{6+n}, q^{6-n}, q^{1-n}, q^{1+n} ; q^{6}\right)_{\infty}}=0
$$

This completes the proof of the theorem.

As in the proof of Theorem 7.1, we can prove the following result. 
Theorem 7.2 Let $n>1$ be an integer coprime with 6 . Then

$$
\begin{aligned}
& \sum_{k=0}^{(n-1) / 2}[8 k+1] \frac{\left(a q, q / a ; q^{2}\right)_{k}\left(q ; q^{2}\right)_{2 k}\left(q^{-1}, q^{4} ; q^{6}\right)_{k}}{\left(q^{2} ; q^{2}\right)_{2 k}\left(a q^{6}, q^{6} / a ; q^{6}\right)_{k}\left(q^{-1}, q^{4} ; q^{2}\right)_{k}} q^{2 k}
\end{aligned}
$$

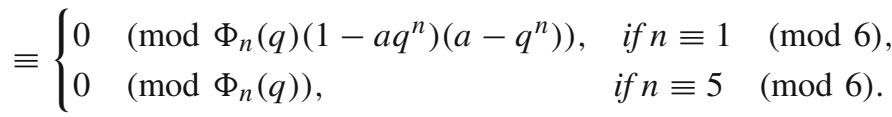

Letting $a \rightarrow 1$ in Theorems 7.1 and 7.2, we obtain

Corollary 7.3 Let $n>1$ be an integer coprime with 6 . Then

$$
\begin{aligned}
& \sum_{k=0}^{(n-1) / 2}[8 k+1] \frac{\left(q ; q^{2}\right)_{k}^{2}\left(q ; q^{2}\right)_{2 k}\left(q, q^{2} ; q^{6}\right)_{k}}{\left(q^{2} ; q^{2}\right)_{2 k}\left(q^{6} ; q^{6}\right)_{k}^{2}(q ; q)_{2 k}} q^{2 k} \\
& \equiv\left\{\begin{array}{lll}
0 & \left(\bmod \Phi_{n}(q)\right), & \text { if } n \equiv 1 \quad(\bmod 6), \\
0 & \left(\bmod \Phi_{n}(q)^{3}\right), & \text { if } n \equiv 5 \quad(\bmod 6),
\end{array}\right.
\end{aligned}
$$

and

$$
\begin{aligned}
& \sum_{k=0}^{(n-1) / 2}[8 k+1] \frac{\left(q ; q^{2}\right)_{k}^{2}\left(q ; q^{2}\right)_{2 k}\left(q^{-1}, q^{4} ; q^{6}\right)_{k}}{\left(q^{2} ; q^{2}\right)_{2 k}\left(q^{6} ; q^{6}\right)_{k}^{2}\left(q^{-1}, q^{4} ; q^{2}\right)_{k}} q^{2 k} \\
& \equiv\left\{\begin{array}{lll}
0 & \left(\bmod \Phi_{n}(q)^{3}\right), & \text { if } n \equiv 1 \quad(\bmod 6), \\
0 & \left(\bmod \Phi_{n}(q)\right), & \text { if } n \equiv 5 \quad(\bmod 6) .
\end{array}\right.
\end{aligned}
$$

We shall also prove the following results.

Theorem 7.4 Let $n$ be a positive integer coprime with 6 . Then

$$
\sum_{k=0}^{M}[8 k+1] \frac{\left(q ; q^{2}\right)_{k}^{2}\left(q ; q^{2}\right)_{2 k}\left(q^{-3} ; q^{6}\right)_{k}}{\left(q^{2} ; q^{2}\right)_{2 k}\left(q^{6} ; q^{6}\right)_{k}\left(q^{-3}, q^{6} ; q^{2}\right)_{k}} q^{2 k} \equiv 0 \quad\left(\bmod [n]^{2}\right),
$$

where $M=n-1$ or $(n-1) / 2$.

Proof It is easy to see by induction on $N$ that

$$
\begin{aligned}
\sum_{k=0}^{N} & \frac{[8 k+1]\left(q ; q^{2}\right)_{k}^{2}\left(q ; q^{2}\right)_{2 k}\left(q^{-3} ; q^{6}\right)_{k}}{\left(q^{2} ; q^{2}\right)_{2 k}\left(q^{6} ; q^{6}\right)_{k}\left(q^{-3}, q^{6} ; q^{2}\right)_{k}} q^{2 k} \\
\quad= & \frac{[4 N+1][4 N+3]\left(q ; q^{2}\right)_{N+1}\left(q ; q^{2}\right)_{2 N}\left(q^{3} ; q^{6}\right)_{N}}{\left(1-q^{3}\right)\left(q^{6} ; q^{6}\right)_{N}\left(q^{2} ; q^{2}\right)_{2 N}\left(q^{6} ; q^{2}\right)_{N}} \\
\quad= & \frac{[4 N+1][4 N+3][2 N+1][2][4]}{[3][2 N+2][2 N+4](-q ; q)_{N}^{2}(-q ; q)_{2 N}^{2}\left(-q^{3} ; q^{3}\right)_{N}^{2}}\left[\begin{array}{c}
2 N \\
N
\end{array}\right]\left[\begin{array}{c}
4 N \\
2 N
\end{array}\right]\left[\begin{array}{c}
2 N \\
N
\end{array}\right]_{q^{3}}
\end{aligned} .
$$


Note that $\frac{1}{[N+1]}\left[\begin{array}{c}2 N \\ N\end{array}\right]$ is the well-known $q$-Catalan number (see [9]), a polynomial in $q$. Hence, the $q$-binomial coefficient $\left[\begin{array}{c}2 N \\ N\end{array}\right]$ is divisible by $[N+1]$, so is $\left[\begin{array}{c}2 N \\ N\end{array}\right]_{q^{3}}$ if $N+1$ is coprime with 3 . It is also not difficult to prove that $\left[\begin{array}{l}4 N \\ 2 N\end{array}\right]$ is divisible by $[N+1]$ whenever $N+1$ is coprime with 6 . Therefore, putting $N=n-1$ in (7.7), we can prove that the right-hand side is congruent to 0 modulo $[n]^{2}$. Similarly, taking $N=(n-1) / 2$ in (7.7), we arrive at the same conclusion. This time one $[n]$ comes from $[2 N+1]$ and another $[n]$ comes from $\left[\begin{array}{l}4 N \\ 2 N\end{array}\right]$.

\section{Some q-Congruences from a Quartic Transformation of Gasper and Rahman}

Gasper and Rahman [12] (see also [13, Ex. 3.33]) also obtained the following quartic transformation:

$$
\begin{aligned}
& \sum_{k=0}^{\infty} \frac{1-a^{2} b^{2} q^{5 k-2}}{1-a^{2} b^{2} / q^{2}} \frac{(a, b ; q)_{k}\left(a b / q, a b, a b q ; q^{3}\right)_{k}\left(a^{2} b^{2} / q^{2} ; q^{4}\right)_{k}}{\left(a b^{2} q^{2}, a^{2} b q^{2} ; q^{4}\right)_{k}\left(a b q, a b, a b / q ; q^{2}\right)_{k}(q ; q)_{k}} q^{k} \\
& \quad=\frac{(a q, b ; q)_{\infty}\left(-a b q ; q^{2}\right)_{\infty}}{(q ; q)_{\infty}\left(b, a b^{2} q^{2}, a^{2} b q^{2} ; q^{4}\right)_{\infty}}{ }_{1} \phi_{1}\left[\begin{array}{c}
a \\
\left.a q^{4} ; q^{4}, b q^{4}\right]
\end{array}\right.
\end{aligned}
$$

In this section, we shall deduce two congruences from the quartic transformation (8.1).

Theorem 8.1 Let $n$ be a positive integer with $n \equiv 5,7(\bmod 8)$. Then

$$
\sum_{k=0}^{(n-1) / 2}[10 k+2] \frac{(q ; q)_{2 k}\left(q ; q^{2}\right)_{3 k}\left(q^{2} ; q^{8}\right)_{k}}{\left(q^{9}, q^{8} ; q^{8}\right)_{k}\left(q ; q^{2}\right)_{2 k}\left(q^{5} ; q^{4}\right)_{k}\left(q^{2} ; q^{2}\right)_{k}} q^{2 k} \equiv 0 \quad\left(\bmod \Phi_{n}(q)\right)
$$

Proof Replacing $q$ by $q^{2}, a$ by $q^{1-n}$, and $b$ by $q^{2-n}$ in (8.1), we see that the left-hand side terminates at $k=(n-1) / 2$, while the right-hand side vanishes. (Note that we cannot make such a replacement if $n \equiv 1,3(\bmod 8)$.) Namely, we have

$$
\sum_{k=0}^{(n-1) / 2} \frac{1-q^{10 k+2-4 n}}{1-q^{2-4 n}} \frac{\left(q^{1-n} ; q\right)_{2 k}\left(q^{1-2 n} ; q^{2}\right)_{3 k}\left(q^{2-4 n} ; q^{8}\right)_{k}}{\left(q^{9-3 n}, q^{8-3 n} ; q^{8}\right)_{k}\left(q^{1-2 n} ; q^{2}\right)_{2 k}\left(q^{5-2 n} ; q^{4}\right)_{k}\left(q^{2} ; q^{2}\right)_{k}} q^{2 k}=0
$$

Since $q^{n} \equiv 1\left(\bmod \Phi_{n}(q)\right)$, we immediately obtain (8.2) from the above identity.

It is not difficult to see that the congruence (8.2) can also be derived from the following quartic summation formula of Gasper [11] (see also [13, Ex. 3.30]):

$$
\begin{gathered}
\sum_{k=0}^{\infty} \frac{1-a q^{5 k}}{1-a} \frac{(a, b ; q)_{k}\left(q / b, q^{2} / b, q^{3} / b ; q^{3}\right)_{k}\left(a^{2} b^{2} / q^{2} ; q^{4}\right)_{k}}{\left(q^{4}, a q^{4} / b ; q^{4}\right)_{k}\left(a b q, a b, a b / q ; q^{2}\right)_{k}\left(q^{3} / a b^{2} ; q\right)_{k}} q^{k} \\
+\frac{a b^{3}(a q, b q, 1 / b ; q)_{\infty}\left(a^{2} b^{2} q^{2} ; q^{4}\right)_{\infty}}{q^{2}\left(a b, q^{3} / a b^{2} ; q\right)_{\infty}\left(a b / q ; q^{2}\right)_{\infty}\left(q^{4}, a b^{3} / q^{2}, a q^{4} / b ; q^{4}\right)_{\infty}}
\end{gathered}
$$




$$
\begin{aligned}
& \times{ }_{1} \phi_{1}\left[\begin{array}{c}
a^{2} b^{2} / q^{2} \\
a^{2} b^{2} q^{2}
\end{array} ; q^{4}, a b^{3} q^{2}\right] \\
& =\frac{\left(a q, a b^{2} / q^{2} ; q\right)_{\infty}}{(a b ; q)_{\infty}\left(a b / q ; q^{2}\right)_{\infty}\left(a q^{4} / b, a b^{3} / q^{2} ; q^{4}\right)_{\infty}} .
\end{aligned}
$$

Theorem 8.2 Let $n$ be a positive integer with $n \equiv 5,7(\bmod 8)$. Then

$$
\sum_{k=0}^{(n-1) / 2}[10 k+4] \frac{\left(q, q^{3} ; q^{2}\right)_{k}\left(q^{2} ; q^{2}\right)_{3 k}\left(q^{4} ; q^{8}\right)_{k}}{\left(q^{11}, q^{9} ; q^{8}\right)_{k}\left(q^{2} ; q^{2}\right)_{2 k}\left(q^{6} ; q^{4}\right)_{k}\left(q^{2} ; q^{2}\right)_{k}} q^{2 k} \equiv 0 \quad\left(\bmod \Phi_{n}(q)\right) .
$$

Proof Replacing $q$ by $q^{2}, a$ by $q^{1-n}$, and $b$ by $q^{3}$ in (8.1), we see that the left-hand side again terminates at $k=(n-1) / 2$, while the right-hand side vanishes. That is,

$$
\sum_{k=0}^{(n-1) / 2} \frac{1-q^{10 k+4-2 n}}{1-q^{4-2 n}} \frac{\left(q^{1-n}, q^{3} ; q^{2}\right)_{k}\left(q^{2-n} ; q^{2}\right)_{3 k}\left(q^{4-2 n} ; q^{8}\right)_{k}}{\left(q^{11-n}, q^{9-2 n} ; q^{8}\right)_{k}\left(q^{2-n} ; q^{2}\right)_{2 k}\left(q^{6-n} ; q^{4}\right)_{k}\left(q^{2} ; q^{2}\right)_{k}} q^{2 k}=0
$$

The proof of (8.3) then follows from the above identity and the fact $q^{n} \equiv 1$ $\left(\bmod \Phi_{n}(q)\right)$.

We have the following two related conjectures.

Conjecture 8.3 The congruence (8.2) is still true modulo $\Phi_{n}(q)^{2}$ for $n \equiv 5(\bmod 8)$. In particular, if $p \equiv 5(\bmod 8)$, then

$$
\sum_{k=0}^{(p-1) / 2} \frac{(5 k+1)(1)_{2 k}\left(\frac{1}{2}\right)_{3 k}\left(\frac{1}{4}\right)_{k}}{32^{k}\left(\frac{9}{8}\right)_{k}(1)_{k}^{2}\left(\frac{1}{2}\right)_{2 k}\left(\frac{5}{4}\right)_{k}} \equiv 0 \quad\left(\bmod p^{2}\right)
$$

Conjecture 8.4 The congruence (8.3) is still true modulo $\Phi_{n}(q)^{3}$. In particular, if $p \equiv 5,7(\bmod 8)$, then

$$
\sum_{k=0}^{(p-1) / 2} \frac{(5 k+2)\left(\frac{1}{2}\right)_{k}^{2}(1)_{3 k}}{8^{k}\left(\frac{11}{8}\right)_{k}\left(\frac{9}{8}\right)_{k}(1)_{2 k}(1)_{k}} \equiv 0 \quad\left(\bmod p^{3}\right)
$$

\section{Some $q$-Congruences from a New ${ }_{12} \phi_{11}$ Transformation}

In this section, we shall deduce some $q$-congruences from Theorem A.1, a new ${ }_{12} \phi_{11}$ transformation formula, whose proof we give in the appendix. Although all of the $q$-congruences are modulo $\Phi_{n}(q)$, the $q=1$ cases sometimes can be generalized to supercongruences modulo higher powers (see Conjectures 12.6 and 12.7 in the next section). 
Theorem 9.1 Let $n \equiv 1(\bmod 3)$ be an integer and $n>1$. Then

$$
\begin{gathered}
\sum_{k=0}^{(n-1) / 3}[6 k+1] \frac{\left(q ; q^{3}\right)_{k}^{6}\left(q^{3} ; q^{3}\right)_{2 k}}{\left(q^{3} ; q^{3}\right)_{k}^{6}\left(q^{2} ; q^{3}\right)_{2 k}} q^{2 k} \equiv 0 \quad\left(\bmod \Phi_{n}(q)\right), \\
\sum_{k=0}^{(n-1) / 3}[6 k+1] \frac{\left(q ; q^{3}\right)_{k}^{4}\left(q^{3} ; q^{3}\right)_{2 k}}{\left(q^{3} ; q^{3}\right)_{k}^{4}\left(q^{2} ; q^{3}\right)_{2 k}} \equiv 0 \quad\left(\bmod \Phi_{n}(q)\right),
\end{gathered}
$$

and

$$
\sum_{k=0}^{(n-1) / 3}[6 k+1] \frac{\left(q ; q^{3}\right)_{k}^{2}\left(q^{3} ; q^{3}\right)_{2 k}}{\left(q^{3} ; q^{3}\right)_{k}^{2}\left(q^{2} ; q^{3}\right)_{2 k}} q^{-2 k} \equiv 0 \quad\left(\bmod \Phi_{n}(q)\right)
$$

Proof Replacing $q \mapsto q^{3}$ and then letting $a=q^{1-n}, b=c=d=q$ in (A.2), we obtain

$$
\sum_{k=0}^{(n-1) / 3} \frac{1-q^{6 k+1-n}}{1-q^{1-n}} \frac{\left(q^{1-n} ; q^{3}\right)_{k}^{3}\left(q ; q^{3}\right)_{k}^{3}\left(q^{3-n} ; q^{3}\right)_{2 k}}{\left(q^{3-n} ; q^{3}\right)_{k}^{3}\left(q^{3} ; q^{3}\right)_{k}^{3}\left(q^{2-n} ; q^{3}\right)_{2 k}} q^{2 k}=0,
$$

because the right-hand side of (A.2) contains the factor $\left(q^{1-n} ; q^{3}\right)_{\infty}$, which vanishes for $n \equiv 1(\bmod 3)$. Since $q^{n} \equiv 1\left(\bmod \Phi_{n}(q)\right)$, we immediately deduce (9.1a) from (9.2) .

Similarly, if we change $c=q$ to $c \rightarrow 0$ in the above procedure, then we can prove (9.1b), while if we change $c=d=q$ to $c, d \rightarrow 0$ then we are led to (9.1c).

Theorem 9.2 Let $n \equiv 2(\bmod 3)$ be an integer and $n>2$. Then

$$
\begin{gathered}
\sum_{k=0}^{(n+1) / 3}[6 k-1] \frac{\left(q^{-1} ; q^{3}\right)_{k}^{6}\left(q^{3} ; q^{3}\right)_{2 k}}{\left(q^{3} ; q^{3}\right)_{k}^{6}\left(q^{-2} ; q^{3}\right)_{2 k}} q^{4 k} \equiv 0 \quad\left(\bmod \Phi_{n}(q)\right), \\
\sum_{k=0}^{(n+1) / 3}[6 k-1] \frac{\left(q^{-1} ; q^{3}\right)_{k}^{4}\left(q^{3} ; q^{3}\right)_{2 k}}{\left(q^{3} ; q^{3}\right)_{k}^{4}\left(q^{-2} ; q^{3}\right)_{2 k}} \equiv 0 \quad\left(\bmod \Phi_{n}(q)\right),
\end{gathered}
$$

and

$$
\sum_{k=0}^{(n+1) / 3}[6 k-1] \frac{\left(q^{-1} ; q^{3}\right)_{k}^{2}\left(q^{3} ; q^{3}\right)_{2 k}}{\left(q^{3} ; q^{3}\right)_{k}^{2}\left(q^{-2} ; q^{3}\right)_{2 k}} q^{-4 k} \equiv 0 \quad\left(\bmod \Phi_{n}(q)\right)
$$

Proof Replacing $q \mapsto q^{3}$ and then letting $a=q^{-1-n}$ and $b=c=d=q^{-1}$ in (A.2), we obtain

$$
\sum_{k=0}^{(n+1) / 3} \frac{1-q^{6 k-1-n}}{1-q^{-1-n}} \frac{\left(q^{-1-n} ; q^{3}\right)_{k}^{3}\left(q ; q^{3}\right)_{k}^{3}\left(q^{3-n} ; q^{3}\right)_{2 k}}{\left(q^{3-n} ; q^{3}\right)_{k}^{3}\left(q^{3} ; q^{3}\right)_{k}^{3}\left(q^{-2-n} ; q^{3}\right)_{2 k}} q^{4 k}=0
$$


because the right-hand side of (A.2) contains the factor $\left(q^{2-n} ; q^{3}\right)_{\infty}$, which vanishes for $n \equiv 2(\bmod 3)$. It is easy to see that the denominator of $(9.4)$ is relatively prime to $\Phi_{n}(q)$ for $n>2$. Therefore, applying $q^{n} \equiv 1\left(\bmod \Phi_{n}(q)\right)$, we obtain the desired congruence in (9.3a). Similarly (see the proof of (9.1b) and (9.1c)), we can prove (9.3b) and (9.3c).

\section{Some Other $q$-Congruences from the $q$-Dixon Sum}

By using the $q$-Dixon sum [13, Eq. (II.13)],

$$
{ }_{4} \phi_{3}\left[\begin{array}{c}
a,-q \sqrt{a}, b, c \\
-\sqrt{a}, a q / b, a q / c
\end{array} ; q, \frac{q \sqrt{a}}{b c}\right]=\frac{(a q, q \sqrt{a} / b, q \sqrt{a} / c, a q / b c ; q)_{\infty}}{(a q / b, a q / c, q \sqrt{a}, q \sqrt{a} / b c ; q)_{\infty}},
$$

the first author and Zudilin [28, Thm. 4.12] proved the following result.

$$
\begin{gathered}
\sum_{k=0}^{(n-1) / 2} \frac{\left(1+a q^{4 k+1}\right)\left(a^{2} q^{2}, b q^{2}, c q^{2} ; q^{4}\right)_{k}}{(1+a q)\left(a^{2} q^{4} / b, a^{2} q^{4} / c, q^{4} ; q^{4}\right)_{k}}\left(\frac{a q}{b c}\right)^{k} \equiv 0 \\
\left(\bmod \left(1-a^{2} q^{2 n}\right)\right) \quad \text { for } n \equiv 3 \quad(\bmod 4) ;
\end{gathered}
$$

in particular,

$$
\sum_{k=0}^{(n-1) / 2} \frac{\left(1+q^{4 k+1}\right)\left(q^{2} ; q^{4}\right)_{k}^{3}}{(1+q)\left(q^{4} ; q^{4}\right)_{k}^{3}} q^{k} \equiv 0 \quad\left(\bmod \Phi_{n}(q) \Phi_{n}(-q)\right) \quad \text { for } n \equiv 3 \quad(\bmod 4)
$$

They [28, Conj. 4.13] also conjectured that the congruence (10.3) still holds modulo $\Phi_{n}(q)^{2} \Phi_{n}(-q)$.

In this section we shall give further similar congruences from the $q$-Dixon sum.

Theorem 10.1 Let $n \equiv 1(\bmod 4)$ be a positive integer. Then

$$
\sum_{k=0}^{(n+1) / 2} \frac{\left(1+a q^{4 k-1}\right)\left(a^{2} / q^{2}, b / q^{2}, c / q^{2} ; q^{4}\right)_{k}}{\left(1+a q^{-1}\right)\left(a^{2} q^{4} / b, a^{2} q^{4} / c, q^{4} ; q^{4}\right)_{k}}\left(\frac{a q^{7}}{b c}\right)^{k} \equiv 0 \quad\left(\bmod \left(1-a^{2} q^{2 n}\right)\right)
$$

in particular,

$$
\sum_{k=0}^{(n+1) / 2} \frac{\left(1+q^{4 k-1}\right)\left(q^{-2} ; q^{4}\right)_{k}^{3}}{(1+q)\left(q^{4} ; q^{4}\right)_{k}^{3}} q^{7 k} \equiv 0 \quad\left(\bmod \Phi_{n}(q) \Phi_{n}(-q)\right) \text { for } n>1
$$


Proof Letting $q \mapsto q^{4}, a \mapsto a^{2} / q^{2}, b \mapsto b / q^{2}$, and $c \mapsto c / q^{2}$ in (10.1), we get

$$
\begin{aligned}
& \sum_{k=0}^{\infty} \frac{\left(1+a q^{4 k-1}\right)\left(a^{2} / q^{2}, b / q^{2}, c / q^{2} ; q^{4}\right)_{k}}{\left(1+a q^{-1}\right)\left(a^{2} q^{4} / b, a^{2} q^{4} / c, q^{4} ; q^{4}\right)_{k}}\left(\frac{a q^{7}}{b c}\right)^{k} \\
& \quad=\frac{\left(a^{2} q^{2}, a q^{5} / b, a q^{5} / c, a^{2} q^{6} / b c ; q^{4}\right)_{\infty}}{\left(a^{2} q^{4} / b, a^{2} q^{4} / c, a q^{3}, a q^{7} / b c ; q^{4}\right)_{\infty}}
\end{aligned}
$$

Since $n \equiv 1(\bmod 4)$, putting $a= \pm q^{-n}$ in (10.6) we see that the left-hand side terminates at $k=(n+1) / 2$, while the right-hand side vanishes. This proves (10.4). For $n>1$, taking the limit as $a, b, c \rightarrow 1$ in (10.4) we are led to (10.5).

We conjecture that the following stronger version of (10.5) is also true.

Conjecture 10.2 Let $n \equiv 1(\bmod 4)$ be an integer and $n>1$. Then

$$
\sum_{k=0}^{(n+1) / 2} \frac{\left(1+q^{4 k-1}\right)\left(q^{-2} ; q^{4}\right)_{k}^{3}}{(1+q)\left(q^{4} ; q^{4}\right)_{k}^{3}} q^{7 k} \equiv 0 \quad\left(\bmod \Phi_{n}(q)^{2} \Phi_{n}(-q)\right)
$$

Similarly to the proof of Theorem 10.1, taking $q \mapsto q^{4}, a \mapsto a^{2} q^{2}$, and $b=c=$ $q^{-2}$ in (10.1), we can prove the following result.

Theorem 10.3 Let $n>1$ be an odd integer. Then

$$
\sum_{k=0}^{(n-1) / 2} \frac{\left(1+a q^{4 k+1}\right)\left(a^{2} q^{2}, q^{-2}, q^{-2} ; q^{4}\right)_{k}}{(1+a q)\left(a^{2} q^{8}, a^{2} q^{8}, q^{4} ; q^{4}\right)_{k}} a^{9 k} \equiv 0 \quad\left(\bmod \left(1-a^{2} q^{2 n}\right)\right)
$$

in particular,

$$
\sum_{k=0}^{(n-1) / 2} \frac{\left(1+q^{4 k+1}\right)\left(q^{2} ; q^{4}\right)_{k}\left(q^{-2} ; q^{4}\right)_{k}^{2}}{(1+q)\left(q^{8} ; q^{4}\right)_{k}^{2}\left(q^{4} ; q^{4}\right)_{k}} q^{9 k} \equiv 0 \quad\left(\bmod \Phi_{n}(q) \Phi_{n}(-q)\right) .(10.8)
$$

Note that, for $n \equiv 3(\bmod 4)$, we can prove the following three-parametric congruence:

$$
\sum_{k=0}^{(n-1) / 2} \frac{\left(1+a q^{4 k+1}\right)\left(a^{2} q^{2}, b / q^{2}, c / q^{2} ; q^{4}\right)_{k}}{(1+a q)\left(a^{2} q^{8} / b, a^{2} q^{8} / c, q^{4} ; q^{4}\right)_{k}}\left(\frac{a q^{9}}{b c}\right)^{k} \equiv 0 \quad\left(\bmod \left(1-a^{2} q^{2 n}\right)\right)
$$

Besides, for the $q=-1$ case of (10.8), it seems that the corresponding congruence can be strengthened as follows. 
Conjecture 10.4 Let $p \equiv 3(\bmod 4)$. Then

$$
\sum_{k=0}^{(p-1) / 2}(-1)^{k}(4 k+1) \frac{\left(\frac{1}{2}\right)_{k}\left(-\frac{1}{2}\right)_{k}^{2}}{(k+1) !^{2} k !} \equiv 0 \quad\left(\bmod p^{2}\right)
$$

Likewise, performing another set of parameter replacements $q \mapsto q^{4}, a \mapsto a^{2} q^{2}$, $b \mapsto b q^{2}$, and $c \mapsto c / q^{2}$ in (10.1), we can deduce the following result.

Theorem 10.5 Let $n \equiv 3(\bmod 4)$ be a positive odd integer. Then

$$
\sum_{k=0}^{(n-1) / 2} \frac{\left(1+a q^{4 k+1}\right)\left(a^{2} q^{2}, b q^{2}, c / q^{2} ; q^{4}\right)_{k}}{(1+a q)\left(a^{2} q^{4} / b, a^{2} q^{8} / c, q^{4} ; q^{4}\right)_{k}}\left(\frac{a q^{5}}{b c}\right)^{k} \equiv 0 \quad\left(\bmod \left(1-a^{2} q^{2 n}\right)\right)
$$

in particular,

$$
\sum_{k=0}^{(n-1) / 2} \frac{\left(1+q^{4 k+1}\right)\left(q^{2} ; q^{4}\right)_{k}^{2}\left(q^{-2} ; q^{4}\right)_{k}}{(1+q)\left(q^{8} ; q^{4}\right)_{k}\left(q^{4} ; q^{4}\right)_{k}^{2}} q^{5 k} \equiv 0 \quad\left(\bmod \Phi_{n}(q) \Phi_{n}(-q)\right) .
$$

We have the following conjectures.

Conjecture 10.6 For any integer $n \equiv 3(\bmod 4)$ and $n>3$, the congruence $(10.10)$ still holds modulo $\Phi_{n}(q)^{2} \Phi_{n}(-q)$.

Conjecture 10.7 Let $n \equiv 3(\bmod 4)$ be a positive integer. Then

$$
\sum_{k=0}^{(n-1) / 2}[4 k+1] \frac{\left(q^{2} ; q^{4}\right)_{k}\left(q^{4} ; q^{8}\right)_{k}}{\left(q^{4} ; q^{4}\right)_{k}\left(q^{8} ; q^{8}\right)_{k}} q^{k} \equiv 0 \quad\left(\bmod \Phi_{n}(q)^{2} \Phi_{n}(-q)\right)
$$

It is easy to see that the congruence (10.11) is true modulo $\Phi_{n}(q) \Phi_{n}(-q)$ by taking $a, b \rightarrow 1$, and $c \rightarrow-1$ in (10.2). Moreover, it is also true when $q=1$ and $n=p$ is an odd prime, since Tauraso observed that

$$
\sum_{k=0}^{n} \frac{4 k+1}{16^{k}}\left(\begin{array}{c}
2 k \\
k
\end{array}\right)^{2}=\frac{(2 n+1)^{2}}{16^{n}}\left(\begin{array}{c}
2 n \\
n
\end{array}\right)^{2}
$$

While a $q$-analogue of (10.12) was given by the first author [16], namely

$$
\sum_{k=0}^{n} q^{-k}\left[\begin{array}{c}
2 k \\
k
\end{array}\right]^{2}\left(-q^{k+1} ; q\right)_{n-k}^{4}=q^{-n}[2 n+1]^{2}\left[\begin{array}{c}
2 n \\
n
\end{array}\right]^{2}
$$

there is no closed form of the left-hand side of (10.11). 


\section{Some $q$-Congruences from a Double Series Transformation of Ismail, Rahman and Suslov}

In [32, Thm. 1.1] Ismail, Rahman, and Suslov derived the following transformation formula:

$$
\begin{aligned}
\sum_{k=0}^{\infty} & \frac{\left(1-a q^{2 k}\right)(a, b, c, d, e, f ; q)_{k}}{(1-a)(q, a q / b, a q / c, a q / d, a q / e, a q / f ; q)_{k}}\left(\frac{a^{2} q^{2}}{b c d e f}\right)^{k}{ }_{4} \phi_{3}\left[\begin{array}{c}
q^{-k}, a q^{k}, g, h \\
b, c, a g h q / b c
\end{array} ; q, q\right] \\
= & \frac{(a q, a q / d e, a q / d f, a q / e f ; q)_{\infty}}{(a q / d, a q / e, a q / f, a q / \operatorname{def} ; q)_{\infty}} 5 \phi_{4}\left[\begin{array}{c}
a g q / b c, a h q / b c, d, e, f \\
a g h q / b c, a q / b, a q / c, \text { def } / a
\end{array} ; q, q\right] \\
& +\frac{\left(a q, d, e, f, a^{2} q^{2} / b d e f, a^{2} q^{2} / c d e f, a g q / b c, a h q / b c, a^{2} g h q^{2} / b c d e f ; q\right)_{\infty}}{\left(a q / b, a q / c, a q / d, a q / e, a q / f, d e f / a q, a g h q / b c, a^{2} g q^{2} / b c d e f, a^{2} k q^{2} / b c d e f ; q\right)_{\infty}} \\
& \times{ }_{5} \phi_{4}\left[\begin{array}{c}
a g q / d e, a q / d f, a q / e f, a^{2} g q^{2} / b c d e f, a^{2} h q^{2} / b c d e f \\
a^{2} q^{2} / b d e f, a^{2} q^{2} / c d e f, a q^{2} / \text { def }, a^{2} g h q^{2} / b c d e f
\end{array} ;, q\right],
\end{aligned}
$$

provided $\mid a^{2} q^{2} /$ bcdef $\mid<1$.

If in (11.1) we replace $q$ by $q^{3}$, take $a=g=q, h=a q$, and $b=c=d=e=f=$ $q^{2}$, and suitably truncate the sum, then the following "divergent" $q$-supercongruence appears to be true.

Conjecture 11.1 Let $n>1$ be an integer with $n \equiv 1(\bmod 3)$.Then

$$
\sum_{k=0}^{(2 n-2) / 3}[6 k+1] \frac{\left(q ; q^{3}\right)_{k}}{\left(q^{3} ; q^{3}\right)_{k}} q^{-2 k}{ }_{4} \phi_{3}\left[\begin{array}{c}
q^{-3 k}, q^{3 k+1}, q, a q \\
q^{2}, q^{2}, a q^{2}
\end{array} q^{3}, q^{3}\right] \equiv 0 \quad\left(\bmod \Phi_{n}(q)^{2}\right)
$$

Furthermore, the above congruence holds modulo $\Phi_{n}(q)^{3}$ when $a=1$.

On the other hand, if in (11.1) we replace $q$ by $q^{3}$, take $a=g=q^{-1}, h=a q^{-1}$, and $b=c=d=e=f=q$, and suitably truncate the sum, then the following "divergent" $q$-supercongruence appears to be true.

Conjecture 11.2 Let $n>2$ be a positive integer with $n \equiv 2(\bmod 3)$. Then

$$
\begin{aligned}
& \sum_{k=0}^{(2 n-1) / 3}[6 k-1] \frac{\left(q^{-1} ; q^{3}\right)_{k}}{\left(q^{3} ; q^{3}\right)_{k}} q^{-k} \\
& \times{ }_{4} \phi_{3}\left[\begin{array}{c}
q^{-3 k}, q^{3 k-1}, q^{-1}, a q^{-1} \\
q, q, a q^{-2}
\end{array} q^{3}, q^{3}\right] \equiv 0 \quad\left(\bmod \Phi_{n}(q)^{2}\right)
\end{aligned}
$$

Furthermore, the above congruence holds modulo $\Phi_{n}(q)^{3}$ when $a=1$.

If in (11.1) we replace $q$ by $q^{4}$, take $a=b=c=d=e=f=q, g=q^{-1}$, $h=a q^{-1}$, and suitably truncate the sum, then the following $q$-supercongruence appears to be true. 
Conjecture 11.3 Let $n$ be a positive integer with $n \equiv 3(\bmod 4)$. Then

$$
\begin{aligned}
& \sum_{k=0}^{(3 n-1) / 4}[8 k+1] \frac{\left(q ; q^{4}\right)_{k}^{6}}{\left(q^{4} ; q^{4}\right)_{k}^{6}} q^{5 k} \\
& \times{ }_{4} \phi_{3}\left[\begin{array}{c}
q^{-4 k}, q^{4 k+1}, q^{-1}, a q^{-1} \\
q, q, a q
\end{array} ; q^{4}, q^{4}\right] \equiv 0 \quad\left(\bmod [n] \Phi_{n}(q)^{2}\right)
\end{aligned}
$$

Furthermore, the above congruence holds modulo $[n] \Phi_{n}(q)^{3}$ when a $=1$.

On the other hand, if in (11.1) we replace $q$ by $q^{4}$, take $a=b=c=d=e=$ $f=q^{-1}, g=q^{-3}, h=a q^{-3}$, and suitably truncate the sum, then the following $q$-supercongruence appears to be true.

Conjecture 11.4 Let $n>1$ be a positive integer with $n \equiv 1(\bmod 4)$. Then

$$
\begin{aligned}
& \sum_{k=0}^{(3 n+1) / 4}[8 k-1] \frac{\left(q^{-1} ; q^{4}\right)_{k}^{6}}{\left(q^{4} ; q^{4}\right)_{k}^{6}} q^{11 k} \\
& \times{ }_{4} \phi_{3}\left[\begin{array}{c}
q^{-4 k}, q^{4 k-1}, q^{-3}, a q^{-3} \\
\left.q^{-1}, q^{-1}, a q^{-1}, q^{4}\right] \equiv 0 \quad\left(\bmod [n] \Phi_{n}(q)^{2}\right)
\end{array}\right.
\end{aligned}
$$

Furthermore, the above congruence holds modulo $[n] \Phi_{n}(q)^{3}$ when $a=1$.

Ismail, Rahman, and Suslov [32, Eq. (5.4)] also noted the following transformation formula (which can be obtained from (11.1) by taking $d=a q / c$ and $h=0$ ):

$$
\begin{aligned}
\sum_{k=0}^{\infty} & \frac{\left(1-a q^{2 k}\right)(a, b, e, f ; q)_{k}}{(1-a)(q, a q / b, a q / e, a q / f ; q)_{k}}\left(\frac{a q}{b e f}\right)^{k}{ }_{3} \phi_{2}\left[\begin{array}{c}
q^{-k}, a q^{k}, g \\
b, c
\end{array} ;, q\right] \\
= & \frac{(a q, c / e, c / f, a q / e f ; q)_{\infty}}{(c, a q / e, a q / f, c / e f ; q)_{\infty}} 3 \phi_{2}\left[\begin{array}{c}
a g q / b c, e, f \\
e f q / c, a q / b
\end{array} ; q, q\right] \\
& \quad+\frac{(a q, e, f, a c q / b e f, a q / e f, a g q / b c ; q)_{\infty}}{(c, a q / b, a q / e, a q / f, e f / c, a q g / b e f ; q)_{\infty}} 3 \phi_{2}\left[\begin{array}{c}
c / e, c / f, a g q / b e f \\
a c q / b e f, c q / e f
\end{array} ; q, q\right]
\end{aligned}
$$

If in (11.2) we replace $q$ by $q^{4}$, take $a=b=c=e=f=q^{-2}, g=q^{5}$, and truncate the sum, then the following $q$-supercongruence appears to be true.

Conjecture 11.5 Let $n$ be a positive integer with $n \equiv 3(\bmod 8)$. Then

$$
\begin{aligned}
& \sum_{k=0}^{(n+1) / 2}[8 k-2] \frac{\left(q^{-2} ; q^{4}\right)_{k}^{4}}{\left(q^{4} ; q^{4}\right)_{k}^{4}} q^{8 k} \\
& \times{ }_{3} \phi_{2}\left[\begin{array}{c}
q^{-4 k}, q^{4 k-2}, q^{5} \\
q^{-2}, q^{-2}
\end{array} q^{4}, q^{4}\right] \equiv 0 \quad\left(\bmod \Phi_{n}(q)^{2} \Phi_{n}(-q)\right)
\end{aligned}
$$


As before, we can show that all the congruences in Conjectures 11.1-11.5 are true modulo $\Phi_{n}(q)$. For example, we have the following parametric generalization of the congruence (11.3) modulo $\Phi_{n}(q) \Phi_{n}(-q)$.

Theorem 11.6 Let $n$ be a positive integer with $n \equiv 3(\bmod 8)$. Then, modulo $\Phi_{n}(q) \Phi_{n}(-q)$

$$
\sum_{k=0}^{(n+1) / 2}[8 k-2] \frac{\left(q^{-2} ; q^{4}\right)_{k}^{2}\left(a q^{-2}, q^{-2} / a ; q^{4}\right)_{k}}{\left(q^{4} ; q^{4}\right)_{k}^{2}\left(a q^{4}, q^{4} / a ; q^{4}\right)_{k}} q^{8 k}{ }_{3} \phi_{2}\left[\begin{array}{c}
q^{-4 k}, q^{4 k-2}, q^{5} \\
q^{-2}, q^{-2}
\end{array} q^{4}, q^{4}\right] \equiv 0
$$

Proof Let $q \rightarrow q^{4}, a=q^{-2-2 n}, b=c=q^{-2}, e=a q^{-2}$, and $f=q^{-2} / a$ in (11.2). Then the left-hand side terminates at $k=(n+1) / 2$ because of the factor $\left(q^{-2-2 n} ; q^{4}\right)_{k}$ in the numerator, while the right-hand side vanishes because of the factor $\left(q^{2-2 n} ; q^{4}\right)_{\infty}$. The described specialization thus yields the following identity:

$$
\begin{aligned}
& \sum_{k=0}^{(n+1) / 2}\left(1-q^{8 k-2-2 n}\right) \frac{\left(q^{-2-2 n}, q^{-2}, a q^{-2}, q^{-2} / a ; q^{4}\right)_{k}}{\left(q^{4}, q^{4-2 n}, a q^{4-2 n}, q^{4-2 n} / a ; q^{4}\right)_{k}} q^{(8-2 n) k} \\
& \quad \times{ }_{3} \phi_{2}\left[\begin{array}{c}
\left.q^{-4 k}, q^{4 k-2-2 n}, q^{5} ; q^{4}, q^{4}\right]=0 . \\
q^{-2}, q^{-2}
\end{array}\right]
\end{aligned}
$$

Since $q^{2 n} \equiv 1\left(\bmod \Phi_{n}(q) \Phi_{n}(-q)\right)$, we immediately deduce the desired congruence from the above identity.

\section{Concluding Remarks and Further Open Problems}

Most of the congruences in the manuscript [28] are modulo $[n]\left(1-a q^{n}\right)\left(a-q^{n}\right)$. However, the congruence (3.2) does not hold modulo $[n]\left(1-a q^{n}\right)\left(a-q^{n}\right)$ in general. We only have a generalization of (3.2) with $a=1$.

It is easy to see that the following generalization of (2.1b) in Theorem 2.1 is true.

Theorem 12.1 Let $n$ be a positive odd integer. Then, modulo $[n] \Phi_{n}(q)^{2}$,

$$
\begin{aligned}
& \sum_{k=0}^{(n-1) / 2}[4 k+1] \frac{\left(b q ; q^{2}\right)_{k}\left(q ; q^{2}\right)_{k}^{5}}{\left(q^{2} / b ; q^{2}\right)_{k}\left(q^{2} ; q^{2}\right)_{k}^{5}}\left(\frac{q}{b}\right)^{k} \\
& \equiv[n] q^{(1-n) / 2} \sum_{k=0}^{(n-1) / 2} \frac{\left(q / b ; q^{2}\right)_{k}\left(q ; q^{2}\right)_{k}^{3}}{\left(q^{2} / b ; q^{2}\right)_{k}\left(q^{2} ; q^{2}\right)_{k}^{3}} q^{2 k} .
\end{aligned}
$$

Letting $a=1$ in Theorem 3.3, we see that the congruence (12.1) holds modulo $\Phi_{n}(q)^{3}$. Therefore, Theorem 12.1 is equivalent to the left-hand side of (12.1) being congruent to 0 modulo $[n]$. By (3.2), we see that the left-hand side of (12.1) is congruent to 0 modulo $\Phi_{n}(q)$. And the same technique to prove congruences modulo $[n]$ from congruences modulo $\Phi_{n}(q)$ as used in the proofs of (3.7a) and (3.7b) still works here. 
We conjecture that the following generalization of the second part of Theorem 2.3 is true.

Conjecture 12.2 Let $n$ be a positive integer with $n \equiv 2(\bmod 3)$. Then

$$
\sum_{k=0}^{n-1}[6 k+1] \frac{\left(q ; q^{3}\right)_{k}^{6}}{\left(q^{3} ; q^{3}\right)_{k}^{6}} q^{3 k} \equiv 0 \quad\left(\bmod [n] \Phi_{n}(q)^{3}\right)
$$

We also have the following similar conjecture.

Conjecture 12.3 Let $n>1$ be a positive integer with $n \equiv 1(\bmod 3)$. Then

$$
\sum_{k=0}^{n-1}[6 k-1] \frac{\left(q^{-1} ; q^{3}\right)_{k}^{6}}{\left(q^{3} ; q^{3}\right)_{k}^{6}} q^{9 k} \equiv 0 \quad\left(\bmod [n] \Phi_{n}(q)^{3}\right)
$$

Note that, similar to the proof of Theorem 2.3, we can show that the above congruence holds modulo $[n] \Phi_{n}(q)$. We point out that $q$-congruences modulo $[n] \Phi_{n}(q)^{3}$ or $\Phi_{n}(q)^{4}$ are very difficult to prove. As far as we know, the following result

$$
\begin{aligned}
& \sum_{k=0}^{(n-1) / 2}[4 k+1] \frac{\left(q ; q^{2}\right)_{k}^{4}}{\left(q^{2} ; q^{2}\right)_{k}^{4}} \equiv q^{(1-n) / 2}[n] \\
& +\frac{\left(n^{2}-1\right)(1-q)^{2}}{24} q^{(1-n) / 2}[n]^{3}\left(\bmod [n] \Phi_{n}(q)^{3}\right),
\end{aligned}
$$

due to the first author and Wang [26], is the first $q$-congruence modulo $[n] \Phi_{n}(q)^{3}$ in the literature that is completely proved. It is natural to ask whether there is a complete $q$-analogue of Long's supercongruence (1.4).

Inspired by the $q$-congruences in the previous sections, we shall propose the following conjecture.

Conjecture 12.4 Let $n$ be a positive odd integer. Then

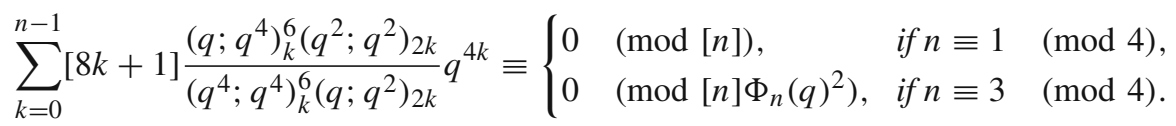

Note that the left-hand side is not a truncated form of (A.2) with $q \mapsto q^{4}$ and $a=$ $b=c=d=q$. Therefore, even for the case modulo $\Phi_{n}(q)$, the above conjecture is still open. Moreover, we cannot find any parametric generalization of the above conjecture, though one would believe that such a generalization should exist.

Similarly, the following conjecture seems to be true.

Conjecture 12.5 Let $n>1$ be an odd integer. Then

$\sum_{k=0}^{n-1}[8 k-1] \frac{\left(q^{-1} ; q^{4}\right)_{k}^{6}\left(q^{2} ; q^{2}\right)_{2 k}}{\left(q^{4} ; q^{4}\right)_{k}^{6}\left(q^{-1} ; q^{2}\right)_{2 k}} q^{8 k} \equiv\left\{\begin{array}{llll}0 & \left(\bmod [n] \Phi_{n}(q)^{2}\right), & \text { if } n \equiv 1 & (\bmod 4), \\ 0 & (\bmod [n]), & \text { if } n \equiv 3 & (\bmod 4)\end{array}\right.$ 
For the $q=1$ case of $(9.1 \mathrm{~b})$, much more seems to be true. Numerical computations suggest the following result.

Conjecture 12.6 Let $p \equiv 1(\bmod 3)$. Then

$$
\sum_{k=0}^{(p-1) / 3}(6 k+1) \frac{\left(\frac{1}{3}\right)_{k}^{4}(2 k) !}{k !^{4}\left(\frac{2}{3}\right)_{2 k}} \equiv p \quad\left(\bmod p^{3}\right)
$$

We also have a similar conjecture related to (9.3b).

Conjecture 12.7 Let $p \equiv 2(\bmod 3)$. Then

$$
\sum_{k=0}^{(p+1) / 3}(6 k-1) \frac{\left(-\frac{1}{3}\right)_{k}^{4}(2 k) !}{k !^{4}\left(-\frac{2}{3}\right)_{2 k}} \equiv p \quad\left(\bmod p^{3}\right)
$$

Unfortunately, we failed to find complete $q$-analogues of the above two conjectures. In particular, we do not know how to use the creative microscoping method to tackle them.

In [16, Conj. 5.4] the first author has made the following conjecture.

Conjecture 12.8 Let $n$ and $r$ be positive integers. Then

$$
\begin{aligned}
& \sum_{k=0}^{n}(-1)^{k} q^{k^{2}+(r-2) k}[4 k+1]\left[\begin{array}{c}
2 k \\
k
\end{array}\right]^{2 r-1}\left(-q^{k+1} ; q\right)_{n-k}^{4 r-2} \equiv 0 \\
& \quad\left(\bmod \left(1+q^{n}\right)^{2 r-2}[2 n+1]\left[\begin{array}{c}
2 n \\
n
\end{array}\right]\right),
\end{aligned}
$$

and

$\sum_{k=0}^{n} q^{(r-2) k}[4 k+1]\left[\begin{array}{c}2 k \\ k\end{array}\right]^{2 r}\left(-q^{k+1} ; q\right)_{n-k}^{4 r} \equiv 0 \quad\left(\bmod \left(1+q^{n}\right)^{2 r-1}[2 n+1]\left[\begin{array}{c}2 k \\ k\end{array}\right]\right)$.

Note that the congruences (12.2a) for $r=1,2$ and (12.2b) for $r=1$ have been proved by the first author [16] himself, and the congruence (12.2b) for $r=2$ has been established by the first author and Wang [26].

In this section, we shall prove the following weaker form of the above conjecture.

Theorem 12.9 The congruences (12.2a) and (12.2b) are true modulo $[n+1][2 n+1]$.

Proof The proof is similar to the second step of that of Theorem 3.3. By Lemma 3.1, it is easy to see that for odd $n$ we have

$$
(-1)^{(n-1) / 2-k} q^{((n-1) / 2-k)^{2}+(r-2)((n-1) / 2-k)} \frac{[2 n-4 k-1]\left(q ; q^{2}\right)_{(n-1) / 2-k}^{2 r-1}}{\left(q^{2} ; q^{2}\right)_{(n-1) / 2-k}^{2 r-1}}
$$




$$
\equiv-(-1)^{k} q^{k^{2}+(r-2) k}[4 k+1] \frac{\left(q ; q^{2}\right)_{k}^{2 r-1}}{\left(q^{2} ; q^{2}\right)_{k}^{2 r-1}} \quad\left(\bmod \Phi_{n}(q)\right),
$$

and

$$
\begin{aligned}
& q^{(r-2)((n-1) / 2-k)} \frac{[2 n-4 k-1]\left(q ; q^{2}\right)_{(n-1) / 2-k}^{2 r}}{\left(q^{2} ; q^{2}\right)_{(n-1) / 2-k}^{2 r}} \\
& \equiv-q^{(r-2) k}[4 k+1] \frac{\left(q ; q^{2}\right)_{k}^{2 r}}{\left(q^{2} ; q^{2}\right)_{k}^{2 r}}\left(\bmod \Phi_{n}(q)\right)
\end{aligned}
$$

This proves that, for odd $n$,

$$
\sum_{k=0}^{(n-1) / 2}(-1)^{k} q^{k^{2}+(r-2) k}[4 k+1] \frac{\left(q ; q^{2}\right)_{k}^{2 r-1}}{\left(q^{2} ; q^{2}\right)_{k}^{2 r-1}} \equiv 0 \quad\left(\bmod \Phi_{n}(q)\right),
$$

and

$$
\sum_{k=0}^{(n-1) / 2} q^{(r-2) k}[4 k+1] \frac{\left(q ; q^{2}\right)_{k}^{2 r}}{\left(q^{2} ; q^{2}\right)_{k}^{2 r}} \equiv 0 \quad\left(\bmod \Phi_{n}(q)\right)
$$

Similar to the proofs of (3.7a) and (3.7b), we can further show that

$$
\sum_{k=0}^{M}(-1)^{k} q^{k^{2}+(r-2) k}[4 k+1] \frac{\left(q ; q^{2}\right)_{k}^{2 r-1}}{\left(q^{2} ; q^{2}\right)_{k}^{2 r-1}} \equiv 0 \quad(\bmod [n]),
$$

and

$$
\sum_{k=0}^{M} q^{(r-2) k}[4 k+1] \frac{\left(q ; q^{2}\right)_{k}^{2 r}}{\left(q^{2} ; q^{2}\right)_{k}^{2 r}} \equiv 0 \quad(\bmod [n]),
$$

where $M=n-1$ or $(n-1) / 2$. It is easy to see that the polynomials $[n+1]$ and $[2 n+1]$ are relatively prime. The proof then follows from the above two congruences by replacing $n$ with $n+1$ or $2 n+1$ and using the relation (1.6).

Finally, we consider the general very-well-poised ${ }_{2 d} \phi_{2 d-1}$ series (which satisfies Slater's transformation [13, $r=d$ and $b_{2 r}=a$ in Eq. (5.5.2)]) where we replace $q$ by $q^{d}$ and take all upper parameters to be $q$. Then the following further generalization of Conjecture 12.2 appears to be true.

Conjecture 12.10 Let $d \geq 3$ and $n$ be positive integers with $n \equiv-1(\bmod d)$. Then

$$
\sum_{k=0}^{M}[2 d k+1] \frac{\left(q ; q^{d}\right)_{k}^{2 d}}{\left(q^{d} ; q^{d}\right)_{k}^{2 d}} q^{d(d-2) k} \equiv 0 \quad\left(\bmod [n] \Phi_{n}(q)^{3}\right),
$$


where $M=((d-1) n-1) / d$ or $n-1$.

Similarly, we consider the general very-well-poised ${ }_{2 d} \phi_{2 d-1}$ series where we replace $q$ by $q^{d}$ and take all upper parameters to be $q^{-1}$. Then the following generalization of Conjecture 12.3 appears to be true.

Conjecture 12.11 Let $d \geq 3$ and $n>1$ be integers with $n \equiv 1(\bmod d)$. Then

$$
\sum_{k=0}^{M}[2 d k-1] \frac{\left(q^{-1} ; q^{d}\right)_{k}^{2 d}}{\left(q^{d} ; q^{d}\right)_{k}^{2 d}} q^{d^{2} k} \equiv 0 \quad\left(\bmod [n] \Phi_{n}(q)^{3}\right),
$$

where $M=((d-1) n+1) / d$ or $n-1$.

Remark Since the submission of the original version of this paper (which also appeared as a preprint on the arXiv) and the present final version, relevant developments have taken place. In particular, some of our conjectures have been confirmed by other authors. Recently, Wang [56] proved Conjecture 6.2 and he further extended it to the modulus $p^{4}$ case. The first author and Zudilin [29] proved Conjecture 10.2, Jana and Kalita [33] proved Conjectures 10.4, 12.6 and 12.7, Ni and Pan [45] proved Conjecture 12.8, and the authors themselves [23,25] confirmed Conjectures 5.4, 12.10 and 12.11. Moreover, Liu [38] generalized Conjectures 12.6 and 12.7 to the modulus $p^{4}$ case.

Acknowledgements The authors would like to thank the anonymous referees for very careful readings of a previous version of this paper. Both authors thank Prof. Shishuo Fu at Chongqing University in China for hosting them for several days in September 2018, during which parts of the present research were performed.

Funding Open access funding provided by University of Vienna.

Open Access This article is licensed under a Creative Commons Attribution 4.0 International License, which permits use, sharing, adaptation, distribution and reproduction in any medium or format, as long as you give appropriate credit to the original author(s) and the source, provide a link to the Creative Commons licence, and indicate if changes were made. The images or other third party material in this article are included in the article's Creative Commons licence, unless indicated otherwise in a credit line to the material. If material is not included in the article's Creative Commons licence and your intended use is not permitted by statutory regulation or exceeds the permitted use, you will need to obtain permission directly from the copyright holder. To view a copy of this licence, visit http://creativecommons.org/licenses/by/4.0/.

\section{Appendix A. A New Nonterminating ${ }_{12} \phi_{11}$ Transformation and Lin- earization of the Continuous $q$-Ultraspherical Polynomials}

Our starting point for deriving the nonterminating transformation formula in Theorem A.1 is the following transformation formula between two terminating very-wellpoised ${ }_{14} \phi_{13}$ series from [35, Thm. 4.1].

$$
\sum_{k=0}^{n} \frac{1-a q^{2 k}}{1-a} \frac{\left(a, b, c, d, a b / c, a b / d, a b q^{n}, q^{-n} ; q\right)_{k}}{\left(q, a q / b, a q / c, a q / d, c q / b, d q / b, q^{1-n} / b, a q^{n+1} ; q\right)_{k}} \frac{(a q / b ; q)_{2 k}}{(a b ; q)_{2 k}}\left(\frac{q}{b}\right)^{2 k}
$$




$$
\begin{aligned}
= & \frac{(a q, \hat{a} q / c, \hat{a} q / d, a q / c d ; q)_{n}}{(\hat{a} q, a q / c, a q / d, \hat{a} q / c d ; q)_{n}} \\
& \times \sum_{k=0}^{n} \frac{1-\hat{a} q^{2 k}}{1-\hat{a}} \frac{\left(\hat{a}, b, c, d, \hat{a} b / c, \hat{a} b / d, \hat{a} b q^{n}, q^{-n} ; q\right)_{k}}{\left(q, \hat{a} q / b, \hat{a} q / c, \hat{a} q / d, c q / b, d q / b, q^{1-n} / b, \hat{a} q^{n+1} ; q\right)_{k}} \frac{(\hat{a} q / b ; q)_{2 k}}{(\hat{a} b ; q)_{2 k}}\left(\frac{q}{b}\right)^{2 k},
\end{aligned}
$$

where $\hat{a}=q^{-n} c d / a b$.

We now have the following new transformation for a nonterminating very-wellpoised ${ }_{12} \phi_{11}$ series into two multiples of nonterminating ${ }_{4} \phi_{3}$ series.

\section{Theorem A.1 We have}

$$
\begin{aligned}
& \sum_{k=0}^{\infty} \frac{1-a q^{2 k}}{1-a} \frac{(a, b, c, d, a b / c, a b / d ; q)_{k}}{(q, a q / b, a q / c, a q / d, c q / b, d q / b ; q)_{k}} \frac{(a q / b ; q)_{2 k}}{(a b ; q)_{2 k}}\left(\frac{q}{b}\right)^{k} \\
& =\frac{(a q, a b / c, a b / d, a q / c d ; q)_{\infty}}{(a b, a q / c, a q / d, a b / c d ; q)_{\infty}} \sum_{k=0}^{\infty} \frac{(b, c, d, c d / a ; q)_{k}}{(q, c q / b, d q / b, c d q / a b ; q)_{k}}\left(\frac{q}{b}\right)^{2 k} \\
& \quad+\frac{\left(a q, c, d, c d q / a b^{2} ; q\right)_{\infty}}{(a b, c q / b, d q / b, c d / a b ; q)_{\infty}} \sum_{k=0}^{\infty} \frac{\left(b, a b / c, a b / d, a b^{2} / c d ; q\right)_{k}}{(q, a q / c, a q / d, a b q / c d ; q)_{k}}\left(\frac{q}{b}\right)^{2 k}
\end{aligned}
$$

where $|q / b|<1$.

This result extends Gasper's [10, Eq. (3.2)] (see also [13, Ex. 8.15]). Observe that the two ${ }_{4} \phi_{3}$ series on the right-hand side are not balanced, nor well-poised. However, they satisfy the remarkable property that the quotient (not the product!) of corresponding upper and lower parameters is throughout the same, namely $b / q$.

By replacing $a, b, c, d$ in (A.2) by $q^{a}, q^{b}, q^{c}, q^{d}$, respectively, and letting $q \rightarrow 1^{-}$ we obtain the following transformation between a nonterminating very-well-poised ${ }_{9} F_{8}$ series into two multiples of nonterminating ${ }_{4} F_{3}$ series. (For the notion of a hypergeometric ${ }_{r} F_{S}$ series, see [2]. In the following, we employ the condensed notation for products of Pochhammer symbols, $\left.\left(a_{1}, \ldots, a_{m}\right)_{k}=\left(a_{1}\right)_{k} \cdots\left(a_{m}\right)_{k}.\right)$

$$
\begin{aligned}
& \sum_{k=0}^{\infty} \frac{a / 2+k}{a / 2} \frac{(a, b, c, d, a+b-c, a+b-d)_{k}}{(1, a+1-b, a+1-c, a+1-d, c+1-b, d+1-b)_{k}} \frac{(a+1-b)_{2 k}}{(a+b)_{2 k}} \\
& =\frac{\Gamma(a+b) \Gamma(a+1-c) \Gamma(a+1-d) \Gamma(a+b-c-d)}{\Gamma(a+1) \Gamma(a+b-c) \Gamma(a+b-d) \Gamma(a+1-c-d)} \\
& \quad \times \sum_{k=0}^{\infty} \frac{(b, c, d, c+d-a)_{k}}{(1, c+1-b, d+1-b, c+d+1-a-b)_{k}} \\
& \quad+\frac{\Gamma(a+b) \Gamma(c+1-b) \Gamma(d+1-b) \Gamma(c+d-a-b)}{\Gamma(a+1) \Gamma(c) \Gamma(d) \Gamma(c+d+1-a-2 b)} \\
& \quad \times \sum_{k=0}^{\infty} \frac{(b, a+b-c, a+b-d, a+2 b-c-d)_{k}}{(1, a+1-c, a+1-d, a+b+1-c-d)_{k}},
\end{aligned}
$$


where, for convergence, $\Re(b)<\frac{3}{4}$.

The transformation in (A.3) extends [10, Eq. (3.3)].

Proof of Theorem A.1 We would like to take $n \rightarrow \infty$ in (A.1) but the series on the righthand side has large terms near the end compared to those in the middle of the series which prevents us from taking the term-by-term limit directly. We thus apply a similar analysis as applied by Bailey [5, Eq. 8.5(3)] in his derivation of the nonterminating Watson transformation (who started with the terminating balanced very-well-poised ${ }_{10} \phi_{9}$ transformation to derive a transformation of a nonterminating very-well-poised ${ }_{8} \phi_{7}$ series into two multiples of balanced ${ }_{4} \phi_{3}$ series), see also [13, Sec. 2.10]. In (A.1), we first replace $n$ by $2 m+1$. Then we write the series on the right-hand side as

$$
\sum_{k=0}^{2 m+1} \lambda_{k}=\sum_{k=0}^{m} \lambda_{k}+\sum_{k=m+1}^{2 m+1} \lambda_{k}=\sum_{k=0}^{m} \lambda_{k}+\sum_{k=0}^{m} \lambda_{2 m+1-k}
$$

after which in each of the sums we can term-wise let $m \rightarrow \infty$ (which is justified by Tannery's theorem [52, p. 292] under the restriction $|q / b|<1$ ). The identity in (A.2) thus follows.

Notice that if in (A.2) we take $d=a / c$ the first series on the right-hand side reduces to 1 . (If instead $d=a b^{2} / c$ then the second series on the right-hand side reduces to 1 . The resulting series is equivalent to (A.5) by the substitution $c \mapsto c / b$.) We thus have the following nonterminating very-well-poised ${ }_{12} \phi_{11}$ summation:

\section{Corollary A.2 We have}

$$
\begin{aligned}
& \sum_{k=0}^{\infty} \frac{\left(1-a q^{2 k}\right)(1-c)(1-a / c)}{(1-a)\left(1-a q^{k} / c\right)\left(1-c q^{k}\right)} \frac{(a, b, b c, a b / c ; q)_{k}}{(q, a q / b, a q / b c, c q / b ; q)_{k}} \frac{(a q / b ; q)_{2 k}}{(a b ; q)_{2 k}}\left(\frac{q}{b}\right)^{k} \\
& =\frac{(q, a q, b c, a b / c ; q)_{\infty}}{(b, c q, a q / c, a b ; q)_{\infty}}+\frac{\left(a q, c, a / c, q / b^{2} ; q\right)_{\infty}}{(a b, c q / b, a q / b c, 1 / b ; q)_{\infty}} \sum_{k=0}^{\infty} \frac{\left(b, b^{2}, b c, a b / c ; q\right)_{k}}{(q, b q, c q, a q / c ; q)_{k}}\left(\frac{q}{b}\right)^{2 k}
\end{aligned}
$$

where $|q / b|<1$.

A further special case of Corollary A.2 is worth stating separately. If we take $b=-1$, the second sum reduces to its first term. We then obtain the following nonterminating very-well-poised ${ }_{12} \phi_{11}$ summation where the two terms on the righthand side satisfy a nice symmetry.

Corollary A.3 We have

$$
\begin{aligned}
& \sum_{k=0}^{\infty} \frac{\left(1-a^{2} q^{4 k}\right)\left(1-c^{2}\right)\left(1-a^{2} / c^{2}\right)}{\left(1-a^{2}\right)\left(1-c^{2} q^{2 k}\right)\left(1-a^{2} q^{2 k} / c^{2}\right)} \frac{(a,-1 ; q)_{k}}{(q,-a q ; q)_{k}}(-q)^{k} \\
& =\frac{(q, a q ; q)_{\infty}}{2(-q,-a ; q)_{\infty}}\left(\frac{(-c,-a / c ; q)_{\infty}}{(c q, a q / c ; q)_{\infty}}+\frac{(c, a / c ; q)_{\infty}}{(-c q,-a q / c ; q)_{\infty}}\right) .
\end{aligned}
$$


If in (A.2) we take (instead of $d=a / c$ which led to Corollary A.2) $d=a q / c$ the prefactor of the first series on the right-hand vanishes. (If instead $d=a b^{2} / c q$ then the prefactor of the second series on the right-hand vanishes. The resulting series is equivalent to (A.7) by the substitution $c \mapsto a b / c$.) We thus have the following nonterminating very-well-poised ${ }_{10} \phi_{9}$ transformation:

Corollary A.4 We have

$$
\begin{aligned}
& \sum_{k=0}^{\infty} \frac{1-a q^{2 k}}{1-a} \frac{(a, b, a b / c, b c / q ; q)_{k}}{\left(q, a q / b, c q / b, a q^{2} / b c ; q\right)_{k}} \frac{(a q / b ; q)_{2 k}}{(a b ; q)_{2 k}}\left(\frac{q}{b}\right)^{k} \\
& =\frac{\left(a q, q^{2} / b^{2}, c, a q / c ; q\right)_{\infty}}{\left(a b, c q / b, a q^{2} / b c, q / b ; q\right)_{\infty}} \sum_{k=0}^{\infty} \frac{\left(b^{2} / q, b c / q, a b / c ; q\right)_{k}}{(q, a q / c, c ; q)_{k}}\left(\frac{q}{b}\right)^{2 k}
\end{aligned}
$$

where $|q / b|<1$.

We also record another (simpler) special case of (A.1), obtained by taking $d \rightarrow \infty$. Alternatively, it can be obtained from Theorem A.1 by choosing $d=q^{-n}$.

Corollary A.5 We have

$$
\begin{aligned}
& \sum_{k=0}^{n} \frac{1-a q^{2 k}}{1-a} \frac{\left(a, b, c, a b / c, a b q^{n}, q^{-n} ; q\right)_{k}}{\left(q, a q / b, a q / c, c q / b, q^{1-n} / b, a q^{n+1} ; q\right)_{k}} \frac{(a q / b ; q)_{2 k}}{(a b ; q)_{2 k}}\left(\frac{q}{b}\right)^{k} \\
& \quad=\frac{(a q, a b / c ; q)_{n}}{(a b, a q / c ; q)_{n}} \sum_{k=0}^{n} \frac{\left(b, c, q^{-n} c / a, q^{-n} ; q\right)_{k}}{\left(q, c q / b, c q^{1-n} / a b, q^{1-n} / b ; q\right)_{k}}\left(\frac{q}{b}\right)^{2 k}
\end{aligned}
$$

We use Corollary A.5, which is equivalent to Gasper's aforementioned identity in [10, Eq. (3.2)] (also stated in [13, Ex. 8.15]), to provide a generalization of Rogers' linearization formula for the continuous $q$-ultraspherical polynomials in (A.12).

The continuous $q$-ultraspherical polynomials, which depend on a parameter $\beta$ and the base $q$, are given by

$$
C_{n}(x ; \beta \mid q)=\sum_{k=0}^{n} \frac{(\beta ; q)_{k}(\beta ; q)_{n-k}}{(q ; q)_{k}(q ; q)_{n-k}} e^{i(n-2 k) \theta}, \quad x=\cos \theta .
$$

(Note that $\theta$ need not be real.) They were originally considered by Rogers [48] in 1884 (not aware of their orthogonality) in the pursuit of (what is now called) the Rogers-Ramanujan identities.

These functions, which can be written as

$$
C_{n}(x ; \beta \mid q)=\frac{(\beta ; q)_{n}}{(q ; q)_{n}} e^{i n \theta}{ }_{2} \phi_{1}\left[\begin{array}{c}
\beta, q^{-n} \\
q^{1-n} / \beta
\end{array} ; q, \frac{q e^{-2 i \theta}}{\beta}\right]
$$

are polynomials in $x$ of degree $n$. 
As was established by Askey and Ismail [4] (see also [31, Thm. 13.2.1]), the continuous $q$-ultraspherical polynomials satisfy for $|\beta|<1$ the orthogonality relation

$$
\begin{aligned}
& \frac{1}{2 \pi} \int_{-1}^{1} C_{m}(x ; \beta \mid q) C_{n}(x ; \beta \mid q) \frac{\left(e^{ \pm 2 i \theta} ; q\right)_{\infty}}{\left(\beta e^{ \pm 2 i \theta} ; q\right)_{\infty}} \frac{\mathrm{d} x}{\sqrt{1-x^{2}}} \\
& \quad=\frac{(\beta, q \beta ; q)_{\infty}}{\left(q, \beta^{2} ; q\right)_{\infty}} \frac{\left(\beta^{2} ; q\right)_{n}}{(q ; q)_{n}} \frac{(1-\beta)}{\left(1-\beta q^{n}\right)} \delta_{m, n} .
\end{aligned}
$$

Rogers [48] (see also [31, Thm. 13.3.2]) derived the following linearization formula:

$$
\begin{aligned}
& C_{m}(x ; \beta \mid q) C_{n}(x ; \beta \mid q) \\
& =\sum_{k=0}^{\min (m, n)} \frac{(q ; q)_{m+n-2 k}(\beta ; q)_{m-k}(\beta ; q)_{n-k}(\beta ; q)_{k}\left(\beta^{2} ; q\right)_{m+n-k}}{\left(\beta^{2} ; q\right)_{m+n-2 k}(q ; q)_{m-k}(q ; q)_{n-k}(q ; q)_{k}(q \beta ; q)_{m+n-k}} \\
& \quad \times \frac{1-\beta q^{m+n-2 k}}{1-\beta} C_{m+n-2 k}(x ; \beta \mid q) .
\end{aligned}
$$

Rogers' proof of (A.12) involved induction. Other proofs have been given by Bressoud [6], Rahman [46], and by Gasper [10] (see also [13, Sec. 8.5]). For our extension of Rogers' linearization formula we define, for $\beta$ and $v$ being any two complex numbers,

$$
F_{v}(z ; \beta \mid q)=\sum_{k \geq 0} \frac{(\beta ; q)_{k}(\beta ; q)_{v-k}}{(q ; q)_{k}(q ; q)_{v-k}} z^{2 k}
$$

which is an even analytic function in $z$. (Recall that in (1.5) the $q$-shifted factorials were defined for any complex subindex.) These functions can be written as

$$
F_{v}(z ; \beta \mid q)=\frac{\left(q^{1+v}, \beta ; q\right)_{\infty}}{\left(q, \beta q^{v} ; q\right)_{\infty}} 2 \phi_{1}\left[\begin{array}{c}
\beta, q^{-v} \\
q^{1-v} / \beta
\end{array} ;, \frac{q z^{2}}{\beta}\right],
$$

where $\left|q z^{2} / \beta\right|<1$, for absolute convergence. For $v=n$ being a nonnegative integer, we have $F_{n}(z ; \beta \mid q)=z^{n} C_{n}(x ; \beta \mid q)$, where $x=\left(z+z^{-1}\right) / 2$ (or, equivalently, $x=\cos \theta$ with $\left.z=e^{i \theta}\right)$.

Theorem A.6 Let $\mu, v$ and $\beta$ be three complex numbers. Then we have the following identity of power series in $z$.

$$
\begin{aligned}
& F_{\mu}(z ; \beta \mid q) F_{v}(z ; \beta \mid q) \\
& =\sum_{k \geq 0} \frac{(q ; q)_{\mu+v-2 k}(\beta ; q)_{\mu-k}(\beta ; q)_{\nu-k}(\beta ; q)_{k}\left(\beta^{2} ; q\right)_{\mu+v-k}}{\left(\beta^{2} ; q\right)_{\mu+v-2 k}(q ; q)_{\mu-k}(q ; q)_{\nu-k}(q ; q)_{k}(q \beta ; q)_{\mu+v-k}} \\
& \quad \times \frac{1-\beta q^{\mu+v-2 k}}{1-\beta} z^{2 k} F_{\mu+v-2 k}(z ; \beta \mid q),
\end{aligned}
$$


where $\left|q z^{2} / \beta\right|<1$, for absolute convergence.

Proof In the subsequent manipulations of series we silently interchange double sums which is justified as all the involved series absolutely converge (if they do not terminate) for $\left|q z^{2} / \beta\right|<1$. We start with expanding the product of the two functions on the lefthand side of (A.15) using (A.13):

$$
\begin{aligned}
& F_{\mu}(z ; \beta, \mid q) F_{\nu}(z ; \beta \mid q) \\
& =\sum_{k \geq 0} \frac{(\beta ; q)_{k}(\beta ; q)_{\mu-k}}{(q ; q)_{k}(q ; q)_{\mu-k}} z^{2 k} \sum_{l \geq 0} \frac{(\beta ; q)_{l}(\beta ; q)_{v-l}}{(q ; q)_{l}(q ; q)_{v-l}} z^{2 l} \\
& =\sum_{k \mapsto k-l} \sum_{l \geq 0} \frac{(\beta ; q)_{k-l}(\beta ; q)_{\mu-k+l}}{(q ; q)_{k-l}(q ; q)_{\mu-k+l}} z^{2 k-2 l} \frac{(\beta ; q)_{l}(\beta ; q)_{v-l}}{(q ; q)_{l}(q ; q)_{v-l}} z^{2 l} \\
& =\sum_{k \geq 0} \sum_{0 \leq l \leq k} \frac{(\beta ; q)_{k-l}(\beta ; q)_{\mu-k+l}}{(q ; q)_{k-l}(q ; q)_{\mu-k+l}} z^{2 k-2 l} \frac{(\beta ; q)_{l}(\beta ; q)_{v-l}}{(q ; q)_{l}(q ; q)_{v-l}} z^{2 l} \\
& =\sum_{k \geq 0} \frac{(\beta ; q)_{k}(\beta ; q)_{\mu-k}}{(q ; q)_{k}(q ; q)_{\mu-k}} \frac{(\beta ; q)_{v}}{(q ; q)_{v}} z^{2 k} \\
& \quad \times \sum_{0 \leq l \leq k} \frac{\left(\beta, q q^{-k}, q^{\mu-k} \beta, q^{-v} ; q\right)_{l}}{\left(q, q^{1-k} / \beta, q^{1+\mu-k}, q^{1-v} / \beta ; q\right)_{l}}\left(\frac{q}{\beta}\right)^{2 l} .
\end{aligned}
$$

Now the inner sum over $l$, which is a special terminating $\phi_{3}$ series, can be transformed into a multiple of a ${ }_{12} \phi_{11}$ series using the $(a, b, c, n) \mapsto\left(\beta q^{\mu+\nu-2 k}, \beta, q^{\mu-k} \beta, k\right)$ case of (A.8). We thus obtain

$$
\begin{aligned}
& F_{\mu}(z ; \beta, \mid q) F_{v}(z ; \beta \mid q) \\
& =\sum_{k \geq 0} \frac{(\beta ; q)_{k}(\beta ; q)_{\mu-k}}{(q ; q)_{k}(q ; q)_{\mu-k}} \frac{(\beta ; q)_{\nu}}{(q ; q)_{\nu}} z^{2 k} \frac{\left(\beta^{2} q^{\mu+\nu-2 k}, q^{1+v-k} ; q\right)_{k}}{\left(\beta q^{1+\mu+\nu-2 k}, \beta q^{\nu-k} ; q\right)_{k}} \\
& \times \sum_{0 \leq l \leq k} \frac{1-\beta q^{\mu+\nu-2 k+2 l}}{1-\beta q^{\mu+\nu-2 k}} \frac{\left(\beta q^{\mu+\nu-2 k}, \beta, \beta q^{\mu-k}, \beta q^{\nu-k}, b a^{2} q^{\mu+\nu-k}, q^{-k} ; q\right)_{l}}{\left(q, q^{1+\mu+\nu-2 k}, q^{1+\nu-k}, q^{1+\mu-k}, q^{1-k} / \beta, \beta q^{1+\mu+\nu-k} ; q\right)_{l}} \\
& \times \frac{\left(q^{1+\mu+\nu-2 k} ; q\right)_{2 l}}{\left(\beta^{2} q^{\mu+\nu-2 k} ; q\right)_{2 l}}\left(\frac{q}{\beta}\right)^{l} \\
& =\sum_{l \geq 0} \sum_{k \geq l} \frac{(\beta ; q)_{k}(\beta ; q)_{\mu-k}}{(q ; q)_{k}(q ; q)_{\mu-k}} \frac{(\beta ; q)_{v}}{(q ; q)_{\nu}} z^{2 k} \frac{\left(\beta^{2} q^{\mu+\nu-2 k}, q^{1+v-k} ; q\right)_{k}}{\left(\beta q^{1+\mu+v-2 k}, \beta q^{\nu-k} ; q\right)_{k}} \frac{1-\beta q^{\mu+\nu-2 k+2 l}}{1-\beta q^{\mu+\nu-2 k}} \\
& \times \frac{\left(\beta q^{\mu+\nu-2 k}, \beta, \beta q^{\mu-k}, \beta q^{\nu-k}, b a^{2} q^{\mu+\nu-k}, q^{-k} ; q\right)_{l}}{\left(q, q^{1+\mu+\nu-2 k}, q^{1+\nu-k}, q^{1+\mu-k}, q^{1-k} / \beta, \beta q^{1+\mu+\nu-k} ; q\right)_{l}} \frac{\left(q^{1+\mu+\nu-2 k} ; q\right)_{2 l}}{\left(\beta^{2} q^{\mu+\nu-2 k} ; q\right)_{2 l}}\left(\frac{q}{\beta}\right)^{l} \\
& \underset{k \mapsto k+l}{=} \sum_{l \geq 0} \sum_{k \geq 0} \frac{(\beta ; q)_{l}(\beta ; q)_{\mu+v-2 k-l}}{(q ; q)_{l}(q ; q)_{\mu+v-2 k-l}} z^{2 l+2 k} \frac{1-\beta q^{\mu+\nu-2 k}}{1-\beta} \\
& \times \frac{(q ; q)_{\mu+\nu-2 k}(\beta ; q)_{\mu-k}(\beta ; q)_{\nu-k}(\beta ; q)_{k}\left(\beta^{2} ; q\right)_{\mu+\nu-k}}{\left(\beta^{2} ; q\right)_{\mu+\nu-2 k}(q ; q)_{\mu-k}(q ; q)_{\nu-k}(q ; q)_{k}(q \beta ; q)_{\mu+\nu-k}}
\end{aligned}
$$




$$
\begin{aligned}
= & \sum_{k \geq 0} \frac{(q ; q)_{\mu+v-2 k}(\beta ; q)_{\mu-k}(\beta ; q)_{\nu-k}(\beta ; q)_{k}\left(\beta^{2} ; q\right)_{\mu+v-k}}{\left(\beta^{2} ; q\right)_{\mu+v-2 k}(q ; q)_{\mu-k}(q ; q)_{\nu-k}(q ; q)_{k}(q \beta ; q)_{\mu+v-k}} \frac{1-\beta q^{\mu+v-2 k}}{1-\beta} z^{2 k} \\
& \times \sum_{l \geq 0} \frac{(\beta ; q)_{l}(\beta ; q)_{\mu+v-2 k-l}}{(q ; q)_{l}(q ; q)_{\mu+v-2 k-l}} z^{2 l},
\end{aligned}
$$

which, after identifying the inner sum as $F_{\mu+\nu-2 k}(z ; \beta \mid q)$ according to (A.13), establishes the assertion.

Corollary A.7 Rogers' linearization formula for the continuous ultraspherical polynomials in (A.12) is true.

Proof In Theorem A. 6 choose $\mu=m$ and $v=n$ for two nonnegative integers $m$ and $n$. The identity (A.15) then reduces, after dividing both sides by $z^{n+m}$, to (A.12).

\section{References}

1. Andrews, G.E.: $q$-Analogs of the binomial coefficient congruences of Babbage, Wolstenholme and Glaisher. Discrete Math. 204, 15-25 (1999)

2. Andrews, G.E., Askey, R.A., Roy, R.: Special functions, Encyclopedia of Mathematics and Its Applications, vol. 71. Cambridge University Press, Cambridge (1999)

3. Ahlgren, S., Ono, K.: Gaussian hypergeometric series evaluation and Apéry number congruences. J. Reine Angew. Math. 518, 187-212 (2000)

4. Askey, R.A., Ismail, M.E.H.: A Generalization of the Ultraspherical Polynomials, in Studies in Pure Mathematics, pp. 55-78. Birkhäuser, Basel (1983)

5. Bailey, W.N.: Generalized Hypergeometric Series. Cambridge University Press, Cambridge (1964)

6. Bressoud, D.: Some identities for terminating $q$-series. Math. Proc. Camb. Phil. Soc. 81, 211-223 (1981)

7. Cao, H.-Q., Pan, H.: Factors of alternating binomial sums. Adv. Appl. Math. 45, 96-107 (2010)

8. Chen, Y.-G., Xie, X.-Y., He, B.: On some congruences of certain binomials sums. Ramanujan J. 40, 237-244 (2016)

9. Fürlinger, J., Hofbauer, J.: q-Catalan numbers. J. Combin. Theory Ser. A 2, 248-264 (1985)

10. Gasper, G.: Rogers' linearization formula for the continuous $q$-ultraspherical polynomials and quadratic transformation formulas. SIAM J. Math. Anal. 16, 1061-1071 (1985)

11. Gasper, G.: Summation, transformation, and expansion formulas for bibasic series. Trans. Am. Math. Soc. 312, 257-277 (1989)

12. Gasper, G., Rahman, M.: An indefinite bibasic summation formula and some quadratic, cubic and quartic summation and transformation formulas. Can. J. Math. 42, 1-27 (1990)

13. Gasper, G., Rahman, M.: Basic Hypergeometric Series. Encyclopedia of Mathematics and Its Applications, vol. 96, 2nd edn. Cambridge University Press, Cambridge (2004)

14. Guillera, J.: WZ pairs and $q$-analogues of Ramanujan series for $1 / \pi$. J. Differ. Equ. Appl. 24, 18711879 (2018)

15. Guillera, J., Zudilin, W.: "Divergent" Ramanujan-type supercongruences. Proc. Am. Math. Soc. 140, 765-777 (2012)

16. Guo, V.J.W.: A $q$-analogue of a Ramanujan-type supercongruence involving central binomial coefficients. J. Math. Anal. Appl. 458, 590-600 (2018)

17. Guo, V.J.W.: A $q$-analogue of the (A.2) supercongruence of Van Hamme for primes $p \equiv 1(\bmod 4)$. Rev. R. Acad. Cienc. Exactas Fís. Nat. Ser. A Mat. 114, Art. 123 (2020)

18. Guo, V.J.W.: q-Analogues of Dwork-type supercongruences. J. Math. Anal. Appl. 487, Art. 124022 (2020)

19. Guo, V.J.W.: q-Analogues of three Ramanujan-type formulas for $1 / \pi$. Ramanujan J. 52, 123-132 (2020)

20. Guo, V.J.W.: $q$-Supercongruences modulo the fourth power of a cyclotomic polynomial via creative microscoping. Adv. Appl. Math. 120, Art. 102078 (2020) 
21. Guo, V.J.W.: $q$-Analogues of two "divergent" Ramanujan-type supercongruences. Ramanujan J. 52, 605-624 (2020)

22. Guo, V.J.W., Schlosser, M.J.: Some new $q$-congruences for truncated basic hypergeometric series. Symmetry 11(2), Art. 268 (2019)

23. Guo, V.J.W., Schlosser, M.J.: Proof of a basic hypergeometric supercongruence modulo the fifth power of a cyclotomic polynomial. J. Differ. Equ. Appl. 25, 921-929 (2019)

24. Guo, V.J.W., Schlosser, M.J.: Some new $q$-congruences for truncated basic hypergeometric series: even powers. Results Math. 75, Art. 1 (2020)

25. Guo, V.J.W., Schlosser, M.J.: A family of $q$-hypergeometric congruences modulo the fourth power of a cyclotomic polynomial. Israel J. Math. (2020). https://doi.org/10.1007/s11856-020-2081-1

26. Guo, V.J.W., Wang, S.-D.: Some congruences involving fourth powers of central $q$-binomial coefficients. Proc. R. Soc. Edinb. Sect. A 150, 1127-1138 (2020)

27. Guo, V.J.W., Zeng, J.: Some $q$-supercongruences for truncated basic hypergeometric series. Acta Arith. 171, 309-326 (2015)

28. Guo, V.J.W., Zudilin, W.: A q-microscope for supercongruences. Adv. Math. 346, 329-358 (2019)

29. Guo, V.J.W., Zudilin, W.: A common $q$-analogue of two supercongruences. Results Math. 75, Art. 46 (2020)

30. Hu, D.W.: On combinatorial congruences and additive combinatorics. Ph.D. thesis, Nanjing University, China (2017)

31. Ismail, M.E.H.: Classical and Quantum Orthogonal Polynomials in One Variable, Encyclopedia of Mathematics and Its Applications, vol. 98. Cambridge University Press, Cambridge (2005)

32. Ismail, M.E.H., Rahman, M., Suslov, S.: Some summation theorems and transformations for $q$-series. Can. J. Math. 49, 543-567 (1997)

33. Jana, A., Kalita, G.: Proof of some conjectural supercongruences of Guo and Schlosser. Ramanujan J. (2020). https://doi.org/10.1007/s11139-019-00221-5

34. Kilbourn, T.: An extension of the Apéry number supercongruence. Acta Arith. 123, 335-348 (2006)

35. Langer, R., Schlosser, M.J., Warnaar, S.O.: Theta functions, elliptic hypergeometric series, and Kawanaka's Macdonald polynomial conjecture. SIGMA 05, 055, p. 20 (2009)

36. Liu, J., Pan, H., Zhang, Y.: A generalization of Morley's congruence. Adv. Differ. Equ. 254, 7 (2015)

37. Liu, J.-C.: On Van Hamme's (A.2) and (H.2) supercongruences. J. Math. Anal. Appl. 471(1-2), 613622 (2019)

38. Liu, J.-C.: Some supercongruences arising from symbolic summation. J. Math. Anal. Appl. 488, Art. $124062(2020)$

39. Liu, J.-C., Petrov, F.: Congruences on sums of $q$-binomial coefficients. Adv. Appl. Math. 116, Art. 102003 (2020)

40. Long, L.: Hypergeometric evaluation identities and supercongruences. Pac. J. Math. 249, 405-418 (2011)

41. Long, L., Ramakrishna, R.: Some supercongruences occurring in truncated hypergeometric series. Adv. Math. 290, 773-808 (2016)

42. Mao, G.-S., Zhang, T.: Proof of Sun's conjectures on super congruences and the divisibility of certain binomial sums. Ramanujan J. 50(1), 1-11 (2019)

43. McCarthy, D., Osburn, R.: A p-adic analogue of a formula of Ramanujan. Arch. Math. 91, 492-504 (2008)

44. Mortenson, E.: A p-adic supercongruence conjecture of Van Hamme. Proc. Am. Math. Soc. 136, 4321-4328 (2008)

45. Ni, H.-X., Pan, H.: Divisibility of some binomial sums. Acta Arith. 194, 367-382 (2020)

46. Rahman, M.: The linearization of the product of the continuous $q$-Jacobi polynomials. Can. J. Math. 33, 255-284 (1981)

47. Rahman, M.: Some quadratic and cubic summation formulas for basic hypergeometric series. Can. J. Math. 45, 394-411 (1993)

48. Rogers, L.J.: Third memoir on the expansion of certain infinite products. Proc. Lond. Math. Soc. 26, 15-32 (1894)

49. Shi, L.-L., Pan, H.: A q-analogue of Wolstenholme's harmonic series congruence. Am. Math. Mon. 114, 529-531 (2005)

50. Straub, A.: Supercongruences for polynomial analogs of the Apéry numbers. Proc. Am. Math. Soc. 147, 1023-1036 (2019)

51. Sun, Z.-W.: Super congruences and Euler numbers. Sci. China Math. 54, 2509-2535 (2011) 
52. Tannery, J.: Introduction a la Théorie des Fonctions d'une Variable, 2nd edn. Tome 1, Libraire Scientifique A. Hermann, Paris (1904)

53. Tauraso, R.: $q$-Analogs of some congruences involving Catalan numbers. Adv. Appl. Math. 48, 603614 (2009)

54. Tauraso, R.: Some $q$-analogs of congruences for central binomial sums. Colloq. Math. 133, 133-143 (2013)

55. Van Hamme, L.: Some conjectures concerning partial sums of generalized hypergeometric series. In: p-Adic Functional Analysis (Nijmegen, 1996), Lecture Notes in Pure and Applied Mathematics, vol. 192, Dekker, New York, pp. 223-236 (1997)

56. Wang, C.: Symbolic summation methods and hypergeometric supercongruences. J. Math. Anal. Appl. 488, Art. 124068 (2020)

57. Wang, X., Yue, M.: A $q$-analogue of the (A.2) supercongruence of Van Hamme for any prime $p \equiv$ 3 ( $\bmod 4)$. Int. J. Number Theory 16, 1325-1335 (2020)

58. Zudilin, W.: Congruences for $q$-binomial coefficients. Ann. Combin. 23, 1123-1135 (2019)

Publisher's Note Springer Nature remains neutral with regard to jurisdictional claims in published maps and institutional affiliations. 